\title{
L'affaire des immunités juridictionnelles de l'Etat (Allemagne c. Italie): la Cour Internationale de Justice à contre-sens de l'évolution du droit international
}

Reçu: 25 novembre 2015

Approuvé: 08 septembre 2017

DOI: dx.doi.org/10.12804/revistas.urosario.edu.co/acdi/a.6538

\section{Paula Wojcikiewicz Almeida*}

Résumé: Cet article vise à évaluer l'affaire des immunités juridictionnelles de l'État devant la Cour Internationale de Justice. Compte tenu de la position adoptée par la majorité des membres de la Cour, le présent article vise à porter un oeil critique sur les arguments qui tendent à considérer qu'il existe un conflit entre l'immunité étatique et les règles de jus cogens,

* Docteur en Droit International et Européen, Université Paris 1 Panthéon-Sorbonne; docteur en Droit International et Intégration Economique, UERJ (co-tutelle); titulaire d'un Master II Recherche en Droit Public International et Européen, Université de Paris XI. Professeur de Droit International à la FGV Droit Rio et chercheuse au Centro de Justiça e Sociedade de la FGV Droit Rio; chercheuse collaboratrice du Module Européen du Programme Jean Monnet, financé par la Commission Européenne à la FGV Droit Rio; chercheuse collaboratrice à l'Institut de Recherche en droit international et européen de la Sorbonne (Iredies); chercheuse du Centre d'Etudes et de Recherches en Droit et Relations Internationales de l'Académie de Droit International de la Haye (2010). Le présent article a été préparé dans le cadre du Cours de perfectionnement pourpraticiens en droit international public et privé de l'Académie de Droit International de la Haye, concernant les immunites en droit international (2013).

Para citar este artículo: Wojcikiewicz Almeida, P., "L’affaire des immunités juridictionnelles de l'Etat (Allemagne c. Italie): la Cour Internationale de Justice à contre-sens de l'évolution du droit international", ACDI-Anuario Colombiano de Derecho Internacional, 2018, 11, pp. 21-70. DoI: dx.doi.org/10.12804/revistas. urosario.edu.co/acdi/a.6538 
en argumentant que la distinction entre les règles de procédure et de fond s'avère être artificielle et formaliste. En outre, l'immunité de l'Etat revêt un caractère relatif lorsque sont en jeu de graves violations des Droits de l'Homme et du Droit International Humanitaire ayant le statut de règles de jus cogens. Il est nécessaire d'effectuer une lecture progressiste de la pratique étatique dans le domaine des immunités étatiques, compte tenu de l'évolution actuelle du Droit International et de l'apparition des individus en tant que sujets de Droit International.

Mots-clés: immunité de l'État, droits de l'homme, jus cogens, affaire des immunités juridictionnelles, Cour Internationale de Justice.

Jurisdictional Immunities of the State (Germany v. Italy): The International Court of Justice against the evolution of International Law

Abstract: The purpose of this article is to assess the jurisdictional immunities of the State case before the International Court of Justice. In view of the position adopted by the majority of the members of the Court, the present article aims at critically examining the arguments that tend to consider that there is a conflict between State immunity and the rules of jus cogens, by arguing that the distinction between procedural and substantive rules is artificial and formalistic. In addition, State immunity is of a relative nature when serious violations of human rights and international humanitarian law, having the status of jus cogens rules, are concerned. It is necessary to carry out a progressive approach of State practice in the field of State immunities, which takes into account the current development of international law and the appearance of individuals as subjects of international law.

Keywords: State immunity, human rights, jus cogens, jurisdictional immunities of the State case, International Court of Justice. 
El caso Inmunidades Jurisdiccionales del Estado (Alemania c. Italia): la Corte Internacional de Justicia en contrasentido de la evolución del Derecho Internacional

Resumen: Este artículo tiene como objetivo evaluar el caso de las inmunidades jurisdiccionales del Estado ante la Corte Internacional de Justicia. Teniendo en cuenta la posición adoptada por la mayoría de los jueces de la Corte, este artículo pretende evaluar críticamente los argumentos que tienden a considerar que existe un conflicto entre la inmunidad del Estado y las normas de jus cogens, argumentando que la distinción entre las reglas de procedimiento y de fondo resulta ser artificial y formalista. Además, la inmunidad del Estado es de carácter relativo cuando están en juego graves violaciones de los derechos humanos y del derecho internacional humanitario con estatus de normas de jus cogens. Es importante hacer una lectura progresista de la práctica de los Estados en el campo de la inmunidad del Estado, dada la evolución actual del derecho internacional y la aparición de los individuos como sujetos de derecho internacional.

Palabras clave: inmunidad del Estado, derechos humanos, jus cogens, caso de las inmunidades jurisdiccionales del Estado, Corte Internacional de Justicia.

\section{Introduction}

L'affaire Allemagne c. Italie auprès de la Cour Internationale de Justice (CIJ) a pour origine des faits survenus pendant la Seconde Guerre Mondiale, lorsque les forces allemandes ont occupé une grande partie du territoire italien, et ont commis de graves violations du Droit International humanitaire. ${ }^{1}$ Figurent parmi ces violations, les massacres de civils et la déportation de civils et militaires pour les soumettre aux travaux forcés en Allemagne et dans les territoires occupés. En février 1947, les Alliés ont conclu un traité de paix avec l'Italie dont le but était de régler les conséquences ju-

\footnotetext{
1 On sait que l'Italie faisait partie de l'Axe, étant alliée de l'Allemagne y compris avant le début de la Seconde Guerre Mondiale. En septembre 1940, l'Allemagne, l'Italie et le Japon ont signé le Pacte Tripartite formant l'Axe. L'Italie s'est seulement retirée de l'Axe en septembre 1943, devenant partie des Alliés. Pour plus de détails, voir Tomuschat, C., "The international law of State immunity and its development by national institutions", Vanderbilt Journal of Transnational Law, 44, p. 1107.
} 
ridiques et économiques de la guerre et, en 1953, la République Fédérale d'Allemagne a adopté une loi fédérale accordant un droit à réparation aux victimes socialistes persécutées par le régime nazi. ${ }^{2}$ Toutefois, différents procès de ressortissants italiens sur le fondement de ladite loi sont restés sans effet en raison de son champ restreint, dont les brèches étaient exploitées de façon à éviter toute sorte d'indemnisation. ${ }^{3}$ Ultérieurement, en 1965, cette loi a été modifiée afin d'élargir son champ d'application, mais elle restait restrictive dans le sens où elle permettait de dédommager seulement des demandeurs ayant obtenu le statut de réfugié au $1^{\text {er }}$ octobre 1953. Pour cette raison, les tribunaux allemands avaient pour habitude de rejeter les demandes en réparation de citoyens italiens fondées sur ladite loi. Deux accords ont été conclus entre l'Allemagne et l'Italie en 1961 par le biais desquels l'Allemagne a accepté de verser une indemnisation à l'Italie et aux citoyens italiens persécutés et directement affectés par les mesures prises par ce pays pendant la guerre. Plus récemment, en août 2000, une loi fédérale a été approuvée, prévoyant un dédommagement indirect des individus soumis, entre autres, aux travaux forcés, à condition qu'ils ne soient pas considérés comme des prisonniers de guerre. Cette restriction a eu pour conséquence que les tribunaux allemands ont privé les citoyens italiens qui auraient été prisonniers de guerre de son bénéfice, même si ce statut n'avait pas été reconnu par le Reich Allemand.

Au vu de ce contexte, Luigi Ferrini, un ressortissant italien qui a été fait prisonnier en août 1944 et déporté vers l'Allemagne, où il a été détenu et soumis aux travaux forcés dans l'industrie de munitions jusqu'à la fin de la guerre, a engagé un procès à l'encontre de l'Allemagne sur le territoire italien, plus précisément auprès du Tribunal d'Arezzo. L'affaire Ferrini constitue la première fois que les tribunaux italiens ont abordé la relation entre l'immunité d'un Etat étranger et les normes relatives aux droits fondamentaux. ${ }^{4}$ Après avoir jugé l'affaire irrecevable en raison de l'immunité de juridiction allemande, la demande de Ferrini a atteint la cour suprême italienne, la Corte di Cassazione. Par un arrêt du 11 mars 2004, ladite Cour

\footnotetext{
2 Bundesentschädigungsgesetz (BEG).

3 Cour Internationale de Justice, Immunités juridictionnelles de l'Etat (Allemagne c. Italie; Grèce intervenant), arrêt du 3 février 2012, \$23.

4 De Sena, P. \& De Vittor, F., "State immunity and human rights: the Italian Supreme Court decision on the Ferrini case", The European Journal of International Law, 2005, 16, (1), p. 90. Pour plus de détails concernant l'affaire voir ibid., pp. 93-95.
} 
a reconnu la compétence des tribunaux italiens en ce qui concerne les demandes en réparation engagées à l'encontre de l'Allemagne par Ferrini, sur le fondement suivant: l'immunité de l'Etat ne s'applique pas lorsque sont en jeu des actes constitutifs de crime international. ${ }^{5}$ De fait, parmi les actes perpétrés par les forces armées allemandes et par d'autres autorités du Reich, figurent les massacres commis à l'encontre de la population civile et la déportation de civils ou prisonniers de guerre vers l'Allemagne afin de les soumettre aux travaux forcés dans l'industrie de l'armement. ${ }^{6}$ Le même fondement a été utilisé par la Cour de Florence dans sa décision de février 2011, dans laquelle il a été décidé que l'Allemagne devrait dédommager Ferrini. D’après la Cour, les règles relatives à l'immunité de juridiction ne seraient pas absolues et ne sauraient être invoquées par un Etat en cas d'actes constituant des crimes au regard du Droit International. L'affaire Ferrini a permis que de nombreuses demandes en réparation soient engagées en Italie par des individus se trouvant ou pas dans des situations similaires, ouvrant la porte à des condamnations de l'Allemagne pour des violations commises pendant la guerre à l'encontre de citoyens italiens et grecs. ${ }^{7}$

5 Ferrini c. República Federal da Alemanba, décision No 5044/2004 (Rivista di diritto internazionale, 2004, 87, p. 539; International Law Reports (ILR), 128, p. 658).

6 La Cour a reconnu que les actes pratiqués par le Reich Allemand violent les considérations élémentaires de l'humanité, citant sa propre jurisprudence dans les affaires du Détroit de Corfou (Royaume-Uni c. Albanie) et Activités Militaires et Paramilitaires au et contre le Nicaragua (Nicaragua c. Etats-unis d'Amérique). Parmi les actes commis, la Cour énumère l'assassinat en grand nombre de civils dans des territoires occupés comme faisant partie d'une politique d'Etat (Civitella in Val di Chiana, Cornia e San Pancrazio); la déportation de membres de la population civile pour les soumettre aux travaux forcés en Allemagne, comme M. Ferrini, qui a été déporté de l'Italie à ces fins; ou encore, la déportation des forces armées italiennes — sans que leur statut de prisonnier de guerre soit reconnu— afin d'accomplir des travaux forcés dans des conditions similaires. Pour la Cour il ne fait aucun doute qu'une telle conduite constitue une grave violation du Droit International des conflits armés applicable en 1943-1945 —Cour Internationale de Justice, Immunités juridictionnelles de l'Etat (Allemagne c. Italie; Grèce intervenant), arrêt du 3 février 2012 (\$ 52 et 94) —, constituant un crime de Droit International ( $(81)$. Les articles 6 b) et 6 c) de la Charte du Tribunal Militaire International de Nuremberg adoptée le 8 août 1945, qui définissent respectivement, les crimes de guerre et les crimes contre l'humanité, corroborent cette affirmation. On sait que les principes de la Charte de Nuremberg ont été ultérieurement confirmés par la résolution de l’Assemblée Générale de l'ONU — résolution 95 (I) du 11 décembre 1946-.

7 Il s'agit du massacre commis par les forces armées allemandes dans la ville grecque de Distomo. Les victimes du massacre ont demandé réparation à l'Allemagne auprès des 
Cherchant à assurer son immunité de juridiction et à éviter l'exécution de ces décisions de condamnation, l'Allemagne a saisi la CIJ, le 23 décembre 2008. Le pays soutenait que l'Italie n'aurait pas respecté l'immunité que lui attribue le Droit International, en jugeant recevables auprès de ses tribunaux des demandes civiles visant à obtenir réparation des dommages causés par des violations du Droit International Humanitaire commises par le Reich allemand pendant la Seconde Guerre Mondiale, ainsi qu'en prenant des mesures d'exécution forcée sur des biens de propriété allemande situés sur le territoire italien (Villa Vigoni). L'Allemagne affirmait également que son immunité aurait été enfreinte par l'Italie en permettant la reconnaissance de décisions de Cours civiles grecques dans des situations similaires. En raison de ces mesures, l'Italie serait responsable internationalement et devrait réparer le préjudice causé.

La Cour a autorisé l'intervention de la Grèce en tant qu'Etat intervenant et a reconnu sa compétence sur le fondement de la clause compromissoire invoquée par l'Allemagne (article $1^{\circ}$ de la Convention Européenne pour le Règlement Pacifique des Différends). Au fond, elle a décidé à la majorité, que l'Italie avait violé l'immunité allemande selon le Droit International, en autorisant des procès civils devant ses tribunaux et en prenant des mesures d'exécution forcée sur des biens dont l'Allemagne était propriétaire, ainsi qu'en faisant reconnaitre des décisions des tribunaux grecs en Italie sur la base des mêmes arguments. En adoptant cette position, qui a déboussolé une bonne partie de la doctrine, ${ }^{8}$ la Cour a

tribunaux grecs, et ultérieurement, auprès de la Cour Européenne des Droits de l'Homme (Cour Européenne des Droits de l'Homme, arrêt du 12 décembre 2012, Kalogeropoulou et autres c. Grèce et Allemagne, requête No 59021/00) et auprès des tribunaux allemands (Citoyens grecs c. République Fédérale de l'Allemagne, affaire No III ZR 245/98). Etant donné que ces tentatives sont restées infructueuses, les demandeurs grecs ont cherché à exécuter les arrêts des tribunaux grecs, qui reconnaissaient leurs droits, auprès des tribunaux italiens. Cf. Cour Internationale de Justice, Immunités juridictionnelles de l'Etat (Allemagne c. Italie; Grèce intervenant), arrêt du 3 février 2012 \$30-35.

8 Bornkamm, C., "State immunity against claims arising from war crimes: the judgement of the International Court of Justice in jurisdictional immunities of the State", German Law Journal, 13, (6), pp. 773-782; Watt, H. M., "Les droits fondamentaux devant les juges nationaux à l'épreuve des immunités juridictionnelles: à propos de l'arrêt de la Cour internationale de justice, immunités juridictionnelles de l'Etat (Allemagne c. Italie; Grèce intervenant), du 3 février 2012", Revue critique de droit international privé, 2012, 101, (3), pp. 539-552; Trapp, K. N. \& Mills, A., "Smooth runs the water where the brook is deep: the obscured complexities of Germany v. Italy", Cambridge Journal of International and 
évité d'affronter l'épineux conflit impliquant l'immunité de l'Etat et la violation des règles de jus cogens, ${ }^{9}$ en rejetant l'idée d'une exception naissante à l'immunité de l'Etat. Au contraire, elle a opté pour une position conservatrice et formaliste, sans prendre en compte les conséquences de

Comparative Law, 2002, 1, pp. 153-168; De Sena \& De Vittor, op. cit., pp. 89-112; Conforti, B., "The judgment of the International Court of Justice on the immunity of foreign States: a missed opportunity", Italian Yearbook of International Law, 2012, XXI, (2011), pp. 135-142; Pavoni, R., "An American anomaly? On the ICJ's selective reading of United States practice in jurisdictional immunities of the State", Italian Yearbook of International Law, 2012, XXI, (2011), pp. 143-159; Espósito, C., "Jus cogens and jurisdictional immunities of States at the International Court of Justice: 'a conflict does exist", Italian Yearbook of International Law, 2012, XXI, (2011), pp. 161-174.

9 Les règles impératives de Droit International ou jus cogens sont des normes supérieures dans l'échelle de la hiérarchie des normes, qui s’imposent y compris contre la volonté des Etats. La notion de jus cogens a été consacrée pour la première fois dans trois articles de la Convention de Vienne sur le Droit des Traités de 1969 (Art. 53, 64 et 71). Une norme impérative ou jus cogens est définie par la convention comme étant "une norme acceptée et reconnue par la communauté internationale des Etats dans son ensemble en tant que norme à laquelle aucune dérogation n'est permise et qui ne peut être modifiée que par une nouvelle norme du droit international général ayant le même caractère". Toutefois, la convention ne mentionne pas l'origine ni le contenu de ces normes, ce qui fait que la jurisprudence internationale intervient progressivement afin d'éclaircir cette notion. Ainsi, la jurisprudence a élargi la notion de jus cogens qui est passée d'une reconnaissance a priori implicite à une consécration explicite. De toute manière, la pratique internationale permet de constater que les normes impératives de Droit International ne se limitent pas au domaine restreint du Droit des Traités, mais s'appliquent au Droit International dans son ensemble. Voir Cour Internationale de Justice, Détroit de Corfou (Royaume-Uni c. Albanie), arrêt du 9 avril 1949 (fond), p. 22; Cour Internationale de Justice, Réserves à la Convention pour la Prévention et la Répression du Crime de Génocide, avis consultatif du 28 mai 1951, p. 22 24; Cour Internationale de Justice, Personnel diplomatique et consulaire des Etats-Unis à Tébéran (Etats-Unis d'Amérique c. Iran), demande d'indication de mesures conservatoires, arrêt du 15 décembre 1979, pp. 18-20, \35-44; Cour Internationale de Justice, Activités militaires et paramilitaires au et contre le Nicaragua (Nicaragua c. Etats-unis d'Amérique), arrêt du 27 juin 196 (fond), pp. 100-101; Cour Internationale de Justice, Licéité de la Menace ou de l'Emploi d'Armes Nucléaires, avis consultatif du 8 juillet 1996, pp. 257-258; Cour Internationale de Justice, Activités armées sur le territoire du Congo (2002) (République Démocratique du Congo c. Rwanda), compétence de la Cour et recevabilité de la demande, arrêt du 3 février 2006, \64-70; TPI (Deuxième Chambre), arrêt du 21 septembre 2005, Ahmed Ali Yusuf et Al Barakaat International Foundation c. Conseil et Commission, affaire T-306/01 et Yassin Abdullah Kadi c. Conseil et Commission, affaire T-315/01; Tribunal Pénal International pour l'ex-Yougoslavie, Chambre de Première Instance, décision du 10 décembre 1998, Procureur contre Anto Furundzija, IT-95-17/1-T, \143-157; Cour Européenne des Droits de l'Homme, arrêt du 21 novembre 2001, Al-Adsani c. Royaume-Uni, requête $\mathrm{N}^{\circ} 35763 / 97$. 
sa décision en ce qui concerne les intérêts des individus lésés, et surtout, en ignorant l'érosion progressive de l'immunité en raison de la limitation de la souveraineté étatique et de l'apparition de l'individu en tant que sujet de Droit International. ${ }^{10}$

Il ne fait aucun doute que les crimes commis constituent de graves violations du Droit International Humanitaire ou du Droit International applicable aux conflits armés de l'époque, ${ }^{11}$ ceux-ci étant entrés dans le domaine du jus cogens. ${ }^{12}$ La présente discussion porte notamment sur l'extension de l'immunité de l'Etat à des cas impliquant la pratique de crimes internationaux caractérisés comme des normes impératives de Droit International. Compte tenu de la position adoptée par la Cour, le présent article vise à porter un oeil critique sur les arguments qui tendent à considérer qu'il existe un conflit entre l'immunité étatique et les règles de jus cogens, en argumentant que la distinction entre les règles de procédure et de fond s'avère être artificielle et formaliste (I). En outre, l'immunité de l'Etat revêt un caractère relatif lorsque sont en jeu de graves violations des Droits de l'Homme et du Droit International Humanitaire ayant le statut de règles de jus cogens (II). Il est nécessaire d'effectuer une lecture progressiste de la pratique étatique dans le domaine des immunités étatiques, compte tenu de l'évolution actuelle du Droit International et de l'apparition des individus en tant que sujets de Droit International. Cela semble avoir été ignoré par la décision de la majorité de la Cour.

10 Dans ce sens, voir Bornkamm, op. cit., p. 778.
11 Cour Internationale de Justice, Immunités juridictionnelles de l'Etat (Allemagne c. Italie; Grèce intervenant), arrêt du 3 février 2012. Cf. article 6 (b) de la Charte du Tribunal Militaire International, 8 août 1945 —United Nations, Treaty Series (UNTS), 82, p. 279— qui définit la notion de crimes de guerre et l'article 6 (c), qui traite des crimes contre l'humanité. Ces principes de la Charte de Nuremberg ont été confirmés par l'Assemblée Générale de l'ONU par le biais de la résolution 95 (I) du 11 décembre 1946.

12 La CIJ s'est prononcée de façon expresse en ce qui concerne l'expression jus cogens dans l'affaire des Activités armées sur le territoire du Congo jugée en 2006 - Cour Internationale de Justice, Activités armées sur le territoire du Congo (2002) (République Démocratique du Congo c. Rwanda), compétence de la Cour et recevabilité de la demande, arrêt du 3 février 2006-. 


\title{
1. L'existence d'un conflit entre l'immunité de l'Etat et les règles de jus cogens
}

\author{
A l'effectivité du ius cogens devant le for national, s'oppose l'artillerie lourde \\ de la logique juridique. ${ }^{13}$
}

La CIJ a rejeté la demande italienne et grecque et a affirmé que l'immunité d'Etat ne pourrait pas être invoquée dans des affaires impliquant la violation de règles impératives du Droit International ou jus cogens. Les deux Etats considéraient qu'une règle impérative écarterait automatiquement toute règle de droit coutumier hiérarchiquement inférieure susceptible d'affecter son application. ${ }^{14}$ Ainsi, il existerait un conflit entre les règles de jus cogens, caractérisées par de graves violations du Droit International Humanitaire, et la règle coutumière qui confère l'immunité à l'Allemagne. Toutefois, selon l'opinion de la Cour, un tel conflit n'existe pas, ${ }^{15}$ car les règles concernant l'immunité de l'Etat revêtent un caractère procédural, liées à l'exercice de la compétence, alors que les normes de Droit International Humanitaire violées (l'interdiction du meurtre, de la déportation et des travaux forcés), qui ont le statut de jus cogens, ont le caractère de règles de fond. Afin de soutenir cet argument, la Cour s'est appuyée sur l'affaire Activités Armées ${ }^{16}$ et sur l'affaire Mandat d'Arrêt, ${ }^{17}$ dans lesquelles elle a considéré que le caractère de jus cogens présent dans les violations de Droit International rapportées n'ont pas pour effet de rendre la Cour compétente, ou d'écarter l'immunité dont le Ministre des Affaires Etrangères

\footnotetext{
13 Watt, H. M., "Les droits fondamentaux devant les juges nationaux à l'épreuve des immunités juridictionnelles: à propos de l'arrêt de la Cour Internationale de Justice", Immunités juridictionnelles de l'Etat (Allemagne c. Italie; Grèce intervenant), du 3 février 2012, p. 6.

14 Comme le prévoit l'article 53 de la Convention de Vienne sur le Droit des Traités de 1969, est considéré comme nul tout traité qui, au moment de sa conclusion, est en conflit avec une norme impérative du droit International général.

15 Cour Internationale de Justice, Immunités juridictionnelles de l'Etat (Allemagne c. Italie; Grèce intervenant), du 3 février 2012, \$93.

16 Cour Internationale de Justice, Activités armées sur le territoire du Congo (2002) (République Démocratique du Congo c. Rwanda), compétence de la Cour et recevabilité de la demande, arrêt du 3 février 2006, \ 64 et 125.

17 Cour Internationale de Justice, Mandat d'Arrêt du 11 avril 2000 (République Démocratique du Congo c. Belgique), arrêt du 14 février 2002, \58 et 78.
} 
bénéficiait en vertu du droit coutumier international. ${ }^{18}$ Des précédents des Cours nationales et de la Cour Européenne des Droits de l'Homme ont également été cités, tels que les affaires $A l$-Adsani c. Royaume-Uni et Kalogeropoulou et autres c. Grèce et Allemagne. ${ }^{19}$

Plusieurs raisons mènent à penser que la position de la Cour ne correspond pas au Droit International contemporain. Le caractère artificiel de la distinction entre les normes de procédure et de fond (1.1) affronte l'effectivité même de l'interdiction impérative établie par la règle de jus cogens (1.2), qui en l'espèce reviendrait à assurer l'impunité de l'Etat infracteur. Des explications tendant à affirmer que l'immunité ne pourrait pas être appliquée à des demandes civiles, mais seulement à des situations impliquant la mise en jeu de la responsabilité pénale s'avèrent également être artificielles et reposent sur des base fragiles (1.3). La prétention d'écarter l'accès à la justice en faveur de l'immunité de l'Etat est encore plus fragile, parce que les deux règles auraient une nature procédurale, sans qu'aucune analyse de la proportionnalité et des intérêts protégés en l'espèce ne soit effectuée (1.4).

\subsection{Le caractère artificiel de la distinction entre les règles de procédure et de fond}

En jugeant l'affaire Allemagne c. Italie, le vote de la majorité a imposé une barrière procédurale à la protection effective des Droits de l'Homme et du Droit International Humanitaire. ${ }^{20}$ Ce n'est pas la première fois que la CIJ établit une distinction entre les normes de fond et procédurales. En effet, dans l'affaire Mandat d'Arrêt, la Cour a affirmé que l'immunité juridictionnelle revêt un caractère procédural, et que la responsabilité pénale a la nature d'une règle de fond. ${ }^{21}$ Toutefois, en l'espèce, il n'a pas été fait

18 Cour Internationale de Justice, Immunités juridictionnelles de l'Etat (Allemagne c. Italie; Grèce intervenant), arrêt du 3 février 2012, \95.

19 Cour Internationale de Justice, Immunités juridictionnelles de l'Etat (Allemagne c. Italie; Grèce intervenant), arrêt du 3 février 2012, \ 96 et 90; Cour Européenne des Droits de l'Homme, arrêt du 21 novembre 2001, Al-Adsani c. Royaume-Uni, requête No 35763/97; Cour Européenne des Droits de l'Homme, arrêt du 12 décembre 2002, Kalogeropoulou et autres c. Grèce et Allemagne, requête $\mathrm{N}^{\circ}$ 59021/00.

20 Dans ce sens, cf. Orakhelashvili, Peremptory norms in international law, Oxford, 2006, pp. 340-341; McGregor, op. cit., pp. 903, 906-907 et 911-912.

21 Cour Internationale de Justice, Mandat d'Arrêt du 11 avril 2000 (République Démocratique du Congo c. Belgique), arrêt du 14 février 2002, \ 60. 
d'analyse critique concernant la distinction entre les normes procédurales et les normes de fond, et les critères de cette distinction n'ont pas été définis. ${ }^{22}$ Le raisonnement de la Cour a été critiqué en grande partie par la doctrine, la décision ayant été considérée comme excessivement formaliste et conservatrice, détachée de la réalité actuelle et ne pouvant pas conduire à des résultats satisfaisants qui prennent en compte les droits des individus. ${ }^{23}$ Cette position semble se fonder sur le propre rôle assumé par la Cour, à savoir, celui d'arbitre neutre des discussions exclusivement interétatiques, détachée des valeurs humanistes qui tissent l'ordre international. ${ }^{24}$ Il s'agit évidemment d'une vision restrictive de sa mission, qui vise à éviter toute interprétation du Droit International susceptible de remettre en question sa légitimité.

Il existe un débat au sein de la doctrine concernant l'existence d'une distinction en Droit International entre les règles qui régissent la procédure, et celles qui régissent le fond, surtout s'agissant des conflits entre l'immunité interétatique et les graves violations des Droits de l'Homme et du Droit International Humanitaire. D'une part, il y a ceux qui soutiennent la nécessité d'une telle distinction dans le cadre du Droit International, afin de justifier la reconnaissance de l'immunité de l'Etat et par conséquent d'écarter les normes impératives violées. ${ }^{25} \mathrm{D}$ 'autre part, il y a ceux

22 La CIJ n’a pas justifié le critère utilisé pour distinguer les règles de fond et procédurales -Talmon, S., "Jus cogens after Germany v. Italy: substantive and procedural rules distinguished", Leiden Journal of International Law, 2012, (25), p. 984- La Cour a seulement estimé que le Droit International, tout comme le droit interne, prévoit cette distinction (idem). Cf. en ce sens Trapp, K. N. \& Mills, A., "Smooth runs the water where the brook is deep: the obscured complexities of Germany v. Italy", Cambridge Journal of International and Comparative Law, 2002, 1, p. 161.

23 Dans ce sens, voir: Bornkamm, op. cit., pp. 773-782; Watt, op. cit., pp. 539-552; De Sena \& De Vittor, op. cit., pp. 89-112; Conforti, B., "The judgment of the International Court of Justice on the immunity of foreign States: a missed opportunity", The Italian Yearbook of International Law, 2012, XXI, (2011), pp. 135-142; Espósito, C., "Jus cogens and jurisdictional immunities of States at the International Court of Justice: 'a conflict does exist", The Italian Yearbook of International Law, 2012, XXI, (2011), pp. 161-174; McGregor, L., "State immunity and jus cogens", International and Comparative Law Quarterly, 2006, 55, (2), pp. 437-445; Pavoni, R., "An American anomaly? On the ICJ's selective reading of United States practice in jurisdictional immunities of the State", Italian Yearbook of International Law, 2012, XXI, (2011), pp. 143-159; Trapp \& Mills, op. cit., pp. 153-168.

24 Watt, op. cit., p. 5.

25 Talmon, op. cit., pp. 979-1002; Tomuschat, C., "L'immunité des Etats en cas de violations graves des droits de l'homme", RGDIP, 2005. 
qui affirment qu'il n'existe pas de catégorie radicale, car il ne s'agit pas de séparer des éléments physiques tels que de l'huile et de l'eau. ${ }^{26}$ Les risques fréquemment pointés du doigt par l'école réaliste ou orthodoxe du Droit International sont la possible détérioration des relations bilatérales entre un Etat territorial et l'Etat responsable de la violation, mais aussi le risque d'innombrables litiges qui fleuriraient auprès des tribunaux nationaux. ${ }^{27}$ Néanmois, ces risques — politiques— ne sont qu'hypothétiques, dépourvus de toute valeur scientifique, et n'ont pas le pouvoir d'influencer le débat autour de la hiérarchie des normes. ${ }^{28}$

\subsection{La garantie de l'effectivité des règles de jus cogens violées}

L'attribution de l'immunité à l'Etat qui a commis de graves violations des Droits de l'Homme et du Droit International Humanitaire finirait par porter atteinte à l'effectivité de la règle de jus cogens, en la vidant de son contenu. Il convient de prendre en compte le contenu de l'ensemble du système normatif, en évitant des contradictions entre les normes. ${ }^{29}$ En effet, il existe des obligations procédurales dérivées du caractère péremptoire des règles de jus cogens. ${ }^{30}$ Cette dimension procédurale trouve son fondement dans les obligations erga omnes de protection issues de la règle de jus cogens en question, comme la Cour l'a elle-même déjà admis dans les affaires Barcelona Traction Light and Power Company Limited (Belgique c. Espagne) et Timor oriental (Portugal c. Australie). ${ }^{31}$ Ainsi toute règle de jus

\footnotetext{
26 Espósito, op. cit., p. 170.

27 Cet argument a été présenté dans l'affaire Al-Adsani c. Royaume-Uni auprès de la Cour Européenne des Droits de l'Homme, arrêt du 21 novembre 2011. Cf. Orakhelashvili, "State immunity and hierarchy of norms: why the House of Lords got it wrong", The European Journal of International Law, 18, (5), p. 956.

28 Orakhelashvili, "State immunity and hierarchy of norms...", ibid., p. 957.

29 Bianchi, A., "Ferrini v. Federal Republic of Germany", The American Journal of International Law, 2005, 99, (1), p. 247.

30 En ce sens, Orakhelashvili, "State immunity and international public order", German Yearbook of International Law, 2002, 45, pp. 227, 258; Bartsch, K. \& Elberling, B., "Jus cogens vs. State immunity, round two: the decision of the European Court of Human Rights in the Kalogeropoulou et al. v. Greece and Germany Decision”, German Law Journal, 2003, 4, (5), pp. 485-488; Espósito, op. cit., pp. 172-173.

31 Voir Cour Internationale de Justice, Barcelona Traction Light and Power Company Limited (Belgique c. Espagne), arrêt du 5 février 1970, \34. Les obligations erga omnes seraient les
} 
cogens impose des obligations erga omnes et vice-versa. ${ }^{32}$ En l'espèce, les obligations erga omnes de protection, qui doivent être garanties horizontalement et verticalement, ${ }^{33}$ consistent à la fois dans l'impossibilité d'invoquer l'immunité de l'Etat en cas de violation de normes impératives et dans la nécessité de dédommager les victimes. ${ }^{34}$

Ainsi, cela n'aurait aucun sens de reconnaittre le statut impératif de la règle d'après le Droit International sans fournir de garantie en vue de son effectivité, en portant atteinte aux droits des individus à obtenir réparation des préjudices qui leur ont été causés en vertu de celles-ci. ${ }^{35}$ En ce sens, l'article 41 du Projet d'articles sur la responsabilité de l'Etat pour fait internationalement illicite, adopté par la Commission de Droit International, qui traite des conséquences de graves violations issues de normes impératives, prévoit que les Etats ne doivent pas reconnaître la licéité d’une

obligations des Etats envers la communauté internationale dans son ensemble. Tous les Etats ont un intérêt juridique à ce que ces droits soient protégés. Voir également Cour Internationale de Justice, Timor Oriental (Portugal c. Australie), arrêt du 30 juin 1995, \2829. D'après le Tribunal Pénal International pour l'ex-Yougoslavie (Chambre de Première Instance, décision du 10 décembre 1998, Procureur contre Anto Furundzija, IT-95-17/1-T, \151), les obligations erga omnes sont des obligations opposables à l'ensemble de la communauté internationale.

32 Cassese, A., "The character of the violated obligation", en Crawford, J., Pellet, A. \& Olleson, S., The law of international responsibility, Oxford University Press, 2010, p. 417.

33 Concernant la double dimension des obligations erga omnes, voir avis consultatif $\mathrm{N}^{\circ}$ 18/03, \ 110 de la Cour Interaméricaine des Droits de l'Homme relatif à la condition juridique des immigrants sans-papiers (Cour Interaméricaine des Droits de l'Homme, avis consultatif $\mathrm{N}^{\circ}$ 18/03 du 17 septembre 2003, Condition Juridique des Immigrants sanspapiers, Série A No 18) et le vote concordant du juge A. A. Cançado Trindade. Tous deux éclaircissent que les obligations erga omnes ont une dimension horizontale, qui s'applique à l'ensemble de la communauté internationale; et une dimension verticale, propre à étendre ces obligations à des organismes et agents publics et particuliers dans leurs relations interindividuelles ( $\$ 76$ et 77 ).

34 En ce sens, voir Trapp \& Mills, op. cit., p. 161. Pour une analyse de cet argument, voir Bartsch \& Elberling, op. cit., p. 487.

35 A l'opposé, certains auteurs considèrent que la nature péremptoire d'une norme n'emporte pas avec soi l'obligation, qui est également issue d'une norme impérative, d'offrir aux victimes des mécanismes afin d'obtenir réparation des dommages provoqués par des actes commis à l'étranger ou par des Etats tiers. En ce sens, voir Tomuschat, "L'immunité des Etats en cas de violations graves des droits de l'homme”, RGDIP, 2005, 109, p. 51; Zimmermann, "Sovereign immunity and violations of international jus cogens. Some critical remarks", Michigan Journal of International Law, 1995, 16, p. 433; Caplan, "State immunity, human rights and jus cogens", AJIL, 2003, 97, p. 741. 
situation engendrée par une violation de norme impérative ni fournir une aide ou une assistance afin de faire perdurer cette situation. ${ }^{36}$ L'obligation de non-reconnaissance renvoie au principe général selon lequel des droits ne peuvent naitre d'un acte illicite (ex injuria jus non oritur). ${ }^{37}$ Son ratio est d'éviter que les violations en question deviennent un fait accompli et se cristallisent dans le temps. ${ }^{38}$ D'après la Commission de Droit International, l'obligation de non-reconnaissance reflète une pratique bien établie et intégrée au sein du Droit International coutumier. ${ }^{39}$ On estime qu'une telle obligation se rapporte à la non-reconnaissance formelle de situation découlant de graves violations, ainsi qu'à l'obligation de ne pas commettre d'actes qui entraîneraient ladite reconnaissance. ${ }^{40}$ Historiquement, l'obligation de non-reconnaissance est liée à l'acquisition de territoire par la force, comme en témoignent les avis consultatifs de la Namibie ${ }^{41}$

36 Bien qu'étant initialement présentes dans le Droit des Traités (article 53 de la Convention de Vienne sur le Droit des Traités de 1969), les règles de jus cogens, après leur reconnaissance jurisprudentielle, se sont étendues au Droit International dans son ensemble. La Commission de Droit International a reconnu l'applicabilité de ces règles en ce qui concerne la responsabilité internationale de l'Etat en raison de violations du Droit International. Voir Commission de Droit International, articles 40-41 sur la Responsabilité de l'Etat, Rapport de la Commission de Droit International (2001), Assemblée Générale, $56^{\text {ème }}$ session, $\mathrm{N}^{\circ}$ 10, (A/56/10). Ainsi, la Commision de Droit International a examiné pour la première fois la question de la relation entre la notion de jus cogens et la responsabilité étatique en 1976 (Ménard, M., "Circumstances precluding wrongfulness in the ILC articles on State responsibility: compliance with peremptory norms", en Crawford, J., Pellet, A. \& Olleson, S., The law of international responsibility, Oxford University Press, 2010, p. 449). Voir également Cassese, A., "The character of the violated obligation", en Crawford, Pellet \& Olleson, ibid., p. 420.

37 En ce sens, voir Cour Permanente Internationale de Justice Internationale, Usine Chrozón, décision du 26 juillet 1927 (juridiction), Série A No 9, p. 4, \31; Cour Internationale de Justice, Conséquences Juridiques pour les Etats de la présence continue de l'Afrique du Sud en Namibie (Sud-ouest africain) nonobstant la résolution 276 (1970) du Conseil de Sécurité, avis consultatif du 21 juin 1971, \46-47.

38 Cour Internationale de Justice, Conséquences juridiques de l'édification d'un mur dans le territoire palestinien occupé, avis consultatif du 9 juillet 2004, \121.

39 Voir commentaire au projet de l'art. 53, \2, ILC Yearbook, 1996, II, (2), \58, 114.

40 Voir Dawidowicz, M., "The obligation of non-recognition of an unlawful situation", en Crawford, Pellet \& Olleson, op. cit., p. 684.

41 Cour Internationale de Justice, Conséquences juridiques pour les Etats de la présence continue de l'Afrique du Sud en Namibie (Sud-ouest africain) nonobstant la résolution 276 (1970) du Conseil de Sécurité, avis consultatif du 21 juin 1971, \53, p. 16; Cour Internationale de Justice, 
et du Mur en Palestine. Ultérieurement, à partir des années 1960, elle a été appliquée à des interdictions d'apartheid, de discrimination raciale, des principes fondamentaux du Droit International Humanitaire et de nonreconnaissance du droit à l'autodétermination.

Le fait que la pratique internationale n'englobe pas explicitement la relation entre l'obligation de non-reconnaissance et les autres normes péremptoires ne contribue pas à écarter son applicabilité au cas concret. Il est évident que l'obligation de non-reconnaissance ne serait pas respectée lorsqu'on accorde l'immunité à un Etat en cas de violation des règles de jus cogens. ${ }^{42} \mathrm{Il}$ serait évidemment contradictoire de reconnaître une grave violation des Droits de l'Homme et du Droit International Humanitaire et, en parallèle, d'accorder l'immunité à celui qui commet ces violations, en refusant aux victimes toute possibité de faire valoir leurs droits. En effet, l'objectif de la règle de jus cogens, en l'espèce, est d'éviter l'impunité. Ainsi, compte tenu des conséquences juridiques issues de la violation d'une règle de jus cogens au regard de la responsabilité de l'Etat, il ne fait aucun doute que l'immunité de l'Etat n'est pas applicable. ${ }^{43}$ Comme le souligne à juste titre M. Espósito, le fait de ne pas permettre aux règles de produire leurs effets maximums et de permettre que des violations restent impunies, est contraire à la nature même des normes péremptoires. ${ }^{44}$

La décision rendue dans l'affaire Procureur c. Furundzija par le Tribunal Pénal pour l'ex-Yougoslavie le 10 décembre 1998 (No IT-95-17/1-T) corrobore le présent argument. La Trial Chamber a considéré que les effets de l'interdiction de la torture, en tant que règle impérative, avaient des répercussions tant dans la sphère interétatique que dans la sphère individuelle. Dans le cadre interétatique, on cherche à ôter la legitimité de toute mesure législative, administrative ou acte judiciaire pouvant permettre la commission de crimes. Ainsi, d'après le tribunal, il serait insensé de considérer, d'un côté, qu'en vertu du caractère obligatoire de l'interdiction de la torture, des traités ou des règles coutumières contraires seraient nuls ab initio (article 53 de la Convention de Vienne sur le Droit des Traités de 1969)

Conséquences juridiques de l'édification d'un mur dans le territoire palestinien occupé, avis consultatif du 9 juillet 2004, p. 136.

42 Orakhelashvili, "State immunity and hierarchy of norms...", op. cit., p. 964.

43 Bianchi, A., "Ferrini v. Federal Republic of Germany", The American Journal of International Law, 2005, 99, (1), p. 247.

44 Espósito, op. cit., p. 172. 
et de permettre d'un autre côté qu'un Etat prenne des mesures nationales qui autorisent ou tolèrent des actes caractérisés par la torture ou absolve ceux qui les ont perpétrés par le biais d'une loi d'amnistie. ${ }^{45}$ Cette logique est transposable en l'espèce dans laquelle un Etat assume internationalement l'obligation de ne pas commettre de graves violations des Droits de l'Homme et du Droit International Humanitaire, que ce soit par le biais de traités ou de la coutume internationale ou, encore en vertu de règles impératives de Droit International. Il serait également insensé d'admettre que l'Etat en question adopte un comportement contradictoire en s'abritant derrière la protection de l'immunité afin de se soustraire aux obligations auxquelles il est tenu en vertu du Droit International. Robert Lecourt, ancien président de la Cour de Justice des Communautés Européennes explique ainsi le problème: toute construction juridique qui permet à un Etat de tirer profit des avantages de la règle commune que les autres sont obligés d'appliquer, mais que cet Etat, si nécessaire, ne l'applique pas de son propre chef serait unique. ${ }^{46}$

Si en théorie, immunité ne siginifie pas impunité, on constate qu'en pratique les deux notions sont intrinsèquement liées l'une à l'autre. Ainsi en accordant l'immunité, on exclut invariablement toute analyse du fond de la question, et en dernière analyse, on corrobore l'idée d'impunité en cas de violation des normes impératives de Droit International. ${ }^{47}$ Comme le souligne à juste titre M. le Juge Van Den Wyngaert, dans son avis dissident dans l'affaire Mandat d'Arrêt, la pratique de l'immunité mène à l'impunité de facto. ${ }^{48}$ Comme la Cour elle-même a semblé le reconnaître en l'espèce, les individus déportés et soumis aux travaux forcés en Allemagne pendant la Seconde Guerre Mondiale, dont la demande constitue le substrat de la présente affaire, n'auront d'autre recours afin de faire valoir

45 Tribunal Pénal International pour l'ex-Yougoslavie, Chambre de Première Instance, décision du 10 décembre 1998, le Procureur contre Anto Furundzija, IT-95-17/1-T, \155.

46 Lecourt, R., L'Europe des juges, Bruylant, Bruselas, 2008 (réimpression de l'oeuvre publiée en 1976, p 285.)

47 Orakhelashvili, "State immunity and hierarchy of norms...", op. cit., pp. 955 et 966967; Espósito, op. cit., p. 172 et note 61. Cour Internationale de Justice, Mandat d'Arrêt du 11 avril 2000 (République Démocratique du Congo c. Belgique), arrêt du 14 février 2002, \$ 60.

48 Opinion dissidente de M. le Juge Van Den Wyngaert, Cour Internationale de Justice, Mandat d'Arrêt du 11 avril 2000 (République Démocratique du Congo c. Belgique), arrêt du 14 février 2002, \ 34. 
leurs droits. ${ }^{49}$ La Cour a seulement fait part de sa surprise et de son regret en constatant que l'Allemagne n'a pas accordé de droit à réparation aux victimes en l'espèce, parce qu'elle ne les considérait pas aptes à cet effet, d'après la loi allemande. ${ }^{50} \mathrm{Il}$ y aurait, dans ce cas, une violation de l'article 41 du Projet de responsabilisation de l'Etat, ${ }^{51}$ car les demandes des victimes dans le cas présent seraient des mesures de last resort, ${ }^{52}$ et s'il n'y est pas fait droit, cela reviendrait à dénier le droit à réparation en raison de la violation d'une norme impérative. ${ }^{53}$ C'est en ce sens que les tribunaux grec et italien ont statué..$^{54}$

En suivant le même raisonnement, les normes internationales qui accordent l'immunité à un Etat infracteur n'auraient aucune reconnaissance juridique. ${ }^{55}$ En effet, la primauté des règles de jus cogens en ce qui concerne l'immunité de l'Etat mènerait à reconnaître que cette dernière est nulle et ne produirait pas d'effets contradictoires avec le contenu de la norme péremptoire violée. ${ }^{56} \mathrm{D}$ 'après le vote dissident des six juges dans l'affaire $A l-A d s a n i$ devant de la Cour Européenne des Droits de l'Homme: un Etat ne peut pas invoquer unilatéralement son immunité dans le but de se soustraire à la mise en jeu de sa responsabilité auprès de juridictions

49 Cour Internationale de Justice, Immunités juridictionnelles de l'Etat (Allemagne c. Italie; Grèce intervenant), arrêt du 3 février 2012, \$ 99. Voir en ce sens, Trapp \& Mills, op. cit., p. 162.

50 Cour Internationale de Justice, Immunités juridictionnelles de l'Etat (Allemagne c. Italie; Grèce intervenant), arrêt du 3 février 2012, \$ 99.

51 En ce sens, Orakhelashvili, "State immunity and hierarchy of norms...", op. cit., p. 970.

52 Dans son opinion dissidente, M. le juge Yusuf a critiqué la décision de la majorité, en affirmant que l'argument du dernier recours a été évalué par la Cour de façon marginale —Opinion dissidente de M. le juge Yusuf, Cour Internationale de Justice Immunités juridictionnelles de l'Etat (Allemagne c. Italie; Grèce intervenant), arrêt du 3 février 2012, \3-.

53 Trapp \& Mills, op. cit., p. 162.

54 Préfecture de Voiotia c. République Fédérale d'Allemagne, Tribunal de Livadia, affaire N ${ }^{\circ}$ 137/1997, décision du 30 octobre 1997. Voir affaire No 137/1997, Massacre de Distomo, Cour collégiale de Livadia, 30 octobre 1997, Revue Hellénique de droit international, 1997, 50, p. 599. Pour plus de détails, voir De Sena, P. \& De Vittor, F., "State immunity and human rights: the Italian Supreme Court decision on the Ferrini case", European Journal of International Law, 2005, 16, (1), p. 89.

55 Tribunal Pénal International pour l'ex-Yougoslavie, Chambre de Première Instance, décision du 10 décembre 1998, Le Procureur contre Anto Furundzija, IT-95-17/1-T, \155.

56 Opinions dissidentes de M. M. les Juges Rozakis, Caflish, Wildhaber, Costa, Cabral Barreto et Vajic, arrêt du 21 novembre 2001, Al-Adsani c. Royaume-Uni, requête No 35763/97, $\int 1$. 
étrangères, en raison d'actes qui constituent des violations de normes impératives de Droit International. ${ }^{57}$ Les règles de jus cogens ont également des conséquences procédurales et ne sauraient être vidées de leur effectivité. ${ }^{58}$ S'il existait une distinction entre procédure et fond, aucun droit fondamental ne serait opposable à un Etat étranger par un individu auprès d'un tribunal national pour des crimes militaires, des actes de barbarie ou tout autre abus officiel, car le statut de jus cogens ne serait pas de nature à modifier les contours de l'immunité souveraine..$^{59}$ Par conséquent, la forme et le fond sont inséparables, contrairement à ce qui ressort de la décision de la Cour dans la présente affaire.

\subsection{Les règles de jus cogens s'appliquent indiféremment en matière civile et pénale}

En troisième lieu, l'argument selon lequel les règles de jus cogens ne seraient applicables qu'en matière pénale reste fragile. ${ }^{60}$ En ce sens, les votes dissidents des juges dans l'affaire $A l$-Adsani ont souligné l'incohérence dans le fait de reconnaittre la primauté des règles impératives en ce qui concerne l'immunité de l'Etat dans des affaires pénales, mais pas dans celles impliquant des demandes civiles. ${ }^{61}$ Ainsi, en accord avec l'opinion dissidente des juges Rozakis, Caflish, Wildhaber, Costa, Cabral Barreto et Vajic, la distinction faite par la majorité concernant les effets des règles de jus cogens (en l'espèce l'interdiction de la torture) dans des procédures civiles et pénales ne correspond pas à l'essence même des règles de jus cogens. D’après les juges, ce n'est pas la nature des procédures qui détermine les

57 Ibid., $\ 1-3$.

58 D'après Trapp \& Mills, op. cit., p. 161: “To suggest that there is no 'inherent' link between the concept of ius cogens and its procedural implications simply avoids the central issue — whether there ought to be such a link — by preconceiving the scope of the norm as a purely substantive question, divorced from any questions of its means of implementation or effectiveness".

59 Watt, op. cit., pp. 7-8.

60 Cour Internationale de Justice, Immunités juridictionnelles de l'Etat (Allemagne c. Italie; Grèce intervenant), arrêt du 3 février 2012, \91.

61 Opinions dissidentes de M. M. les Juges Rozakis, Caflish, Wildhaber, Costa, Cabral Barreto et Vajic, arrêt du 21 novembre 2001, Al-Adsani c. Royaume-Uni, requête No 35763/97, \4. Voir également Orakhelashvili, "State immunity and hierarchy of norms...", op. cit., pp. $965-966$ et 969 . 
effets que la règle de jus cogens aura par rapport à une autre règle de Droit International, mais son caractère péremptoire et son intéraction avec les normes qui lui sont hiérarchiquement inférieures. ${ }^{62}$ Ils concluent que la nature pénale ou civile des procédures nationales n'est pas pertinente. ${ }^{63}$ De fait, les deux sont liés et il n'y a aucune raison valable de considérer que des développements en matière pénale ne doivent pas s’appliquer en matière civile.

Si les règles relatives à l'immunité de l'Etat revêtent un caractère facultatif, on devrait parvenir à la même conclusion dans tous les domaines, que ce soit pénal ou civil, car la discussion du fond est la même: il s'agit d'une contradiction entre les normes impératives et facultatives. ${ }^{64} \mathrm{En}$ outre, les développements dans le domaine pénal pourraient être transposés au domaine civil, en indiquant que de graves violations des Droits de l'Homme et Droit International Humanitaire sont répréhensibles et ne seraient pas soumises à l'immunité. ${ }^{65}$

\subsection{La primauté de l'accès à la justice sur l'immunité de l'Etat}

En quatrième lieu, même si la Cour estimait que l'immunité de l'Etat, en tant que norme de procédure, porterait atteinte à l'examen de la question au fond, à savoir, la grave violation des Droits de l'Homme et du Droit International Humanitaire, cet argument n'empêcherait pas la survenance d'un conflit entre l'immunité de l'Etat et l'accès à la justice des demandeurs. Le droit d'accès à la justice doit être compris lato sensu: c'est le droit que justice soit faite. ${ }^{66} \mathrm{Il}$ s'agit d'un véritable "droit au droit", autrement dit, à un ordre juridique — tant au niveau national qu'international_ qui protège effectivement les droits fondamentaux de l'homme, étant donné

\footnotetext{
$62 \quad$ Ibid., $\$ 4$.

63 Ibid., $\int 4$.

64 Espósito, op. cit., p. 172; Trapp \& Mills, op. cit., p. 161.

65 Pour plus de détails, voir l'opinion dissidente de M. le Juge Van Den Wyngaert, Cour Internationale de Justice, Mandat d'Arrêt du 11 avril 2000 (République Démocratique du Congo c. Belgique), arrêt du 14 février 2002, \ 29 et suiv.

66 Cançado Trindade, A. A., Evolution du droit international au droit des gens - l'accès des individus à la justice internationale, le regard d'un juge, Pedone, París, 2008, p. 81.
} 
que les individus sont les derniers bénéficiaires des droits à préserver. ${ }^{67}$ Progressivement, le droit d'accès à la justice a été reconnu en tant que règle de jus cogens, entraînant avec lui le droit à réparation des victimes de graves violations des Droits de l'Homme. ${ }^{68} \mathrm{Si}$ la réparation individuelle constituait une exception dans le passé, la Commission des Droits de l'Homme de l'ONU travaille actuellement sur ce sujet, l'Assemblée Générale ayant adopté le document intitulé Principes fondamentaux concernant le Droit à un Recours et à Réparation. ${ }^{69}$

Comme cela a été mis en évidence par la Cour Constitutionnelle slovène le 8 mars 2001, ${ }^{70}$ et par la Cour Suprême polonaise le 29 octobre $2010,{ }^{71}$ dont les décisions ont été citées dans l'arrêt de la CIJ, ${ }^{72}$ l'immunité allemande en raison des crimes internationaux n'aurait pu être accordée que s'il y existait un recours alternatif pour les victimes de ces violations. En reprenant les termes du tribunal polonais, l'immunité de l'Etat ne saurait constituer un obstacle irréductible à l'exercice du droit d'accès à la justice des victimes pour protéger leurs droits de façon effective. ${ }^{73}$ Le Tribunal polonais a cité les affaires Waite et Kennedy c. Allemagne et Beer et Regan c. Allemagne de la Cour Européenne des Droits de l'Homme, afin de démontrer la nécessité d'établir un équilibre entre l'immunité et l'accès à la justice. ${ }^{74}$ Comme le souligne M. le juge Yusuf, cette mise en balance doit

67 Cançado Trindade, A. A., Tratado de direito internacional dos direitos bumanos, Tome III, S. A. Fabris, Porto Alegre, Brasil, 2002, p. 524, \187; et Cançado Trindade, A. A., Evolution du droit international au droit des gens..., op. cit., p. 117.

68 La Cour Interaméricaine de Droits de l'Homme a reconnu que le droit d'accès à la justice est entré dans le domaine du jus cogens (Affaires Goiburu et alii. c. Paraguay du 22.09.2006 et La Cantuta c. Pérou du 29.11.2006). Voir l'opinion dissidente de M. le Juge Antônio Augusto Cançado Trindade, S 205-217.

69 GA Res. 60/147, un Doc A/Res/60/147 (16 déc. 2005).

70 Affaire $N^{\circ}$ Up-13/99, Arrêt du 8 mars 2001.

71 Natoniewski c. République Fédérale d'Allemagne, arrêt du 29 octobre 2010, Polish Yearbook. of International Law, 2010, XXX, p. 299.

72 Cour Internationale de Justice, Immunités juridictionnelles de l'Etat (Allemagne c. Italie; Grèce intervenant), arrêt du 3 février 2012, \ 74.

73 Voir Conforti, B., "The judgment of the International Court of Justice on the immunity of foreign States: a missed opportunity”, Italian Yearbook of International Law, 2012, XXI, (2011), p. 139.

74 D'après la Cour Européenne des Droits de l'Homme "[...] a material factor in determining whether granting ESA (the European Space Agency) immunity from German jurisdiction is permissible under the Convention is whether the applicants had available to 
prendre en compte les fonctions et les objectifs intrinsèques de l'immunité, d'une part, et la protection et la réalisation des principes fondamentaux des Droits de l'Homme et Droit Humanitaire, d'autre part. ${ }^{75}$ La Cour aurait dû effectuer une analyse de la proportionnalité de l'objectif d'accorder l'immunité dans des affaires impliquant de graves violations dans lesquelles il n'y avait pas de possibilité de réparation pour les victimes. ${ }^{76}$

Ainsi, en cas de conflit entre l'immunité et l'accès à la justice découlant de violations de règles de jus cogens, il ne fait aucun doute que la balance pencherait vers l'effective réalisation des Droits de l'Homme et du Droit Humanitaire. ${ }^{77}$ En effet, il existerait dans ce cas un conflit entre deux normes de droit procédural, l'accès à la justice revêtant le caractère de règle de jus cogens. ${ }^{78}$ L'accès à la justice serait hiérarchiquement supérieur aux règles coutumières concernant l'immunité juridictionnelle. Il en découle que les arguments contenus dans la décision de la majorité en l'espèce reposent sur des bases fragiles et n'apportent aucune stabilité au

them reasonable alternative means to protect effectively their rights under the Convention" (Cour Européenne des Droits de l'Homme, arrêt du 18 février 1999, Waite et Kennedy c. Allemagne et, requête No 26083/94, \68; et Cour Européenne des Droits de l'Homme, Beer et Regan c. Allemagne, arrêt du 18 février 1999, requête No 28934/95, \58).

75 Opinion dissidente de M. le Juge Yusuf, Immunités juridictionnelles de l'Etat (Allemagne c. Italie; Grèce intervenant), arrêt du 3 février 2012, \$29.

76 Opinion dissidente de M. le Juge Yusuf, Cour Internationale de Justice, Immunités juridictionnelles de l'Etat (Allemagne c. Italie; Grèce intervenant), arrêt du 3 février 2012, \30. Cour Européenne des Droits de l'Homme, arrêt du 18 février 1999, Waite et Kennedy c. Allemagne, requête $\mathrm{N}^{\mathrm{o}} 26083 / 94$, \59; Cour Européenne des Droits de l'Homme, arrêt du 21 novembre 2001, Fogarty c.Royaume-Uni, requête No 37112/97, \33.

77 Conforti, op. cit., p. 136. Vide Thomas \& Small, "Human rights and State immunity: is there immunity from civil liability for torture", NILR, 2003, p. 1; Bianchi, "L'immunité des Etats et les violations graves des droits de l'homme: la fonction de l'interprète dans la détermination du droit international", RGDIP, 2004, p. 63; Focarelli, "Denying foreign State immunity for commission of international crimes: the Ferrini decision", ICLQ, 2005, p. 951; Tomuschat, "L'immunité des Etats en cas de violations graves des droits de l'homme", RGDIP, 2005, p. 194; Sciso, “Italian judges' point of view on foreign States' immunity”, Vanderbilt Journal of Transnational Law, 2011, p. 1201.

78 Watt, op. cit., p. 7. Cour Interaméricaine des Droits de l'Homme, affaires Goiburu et alii c. Paraguay du 22.09.2006 et La Cantuta c. Pérou du 29.11.2006. En ce sens, voir l'opinion dissidente de M. le Juge A. A. Cançado Trindade, Cour Internationale de Justice, Immunités juridictionnelles de l'Etat (Allemagne c. Italie; Grèce intervenant), arrêt du 3 février 2012, \205-217. 
système, contrairement à l'intention de la Cour de se positionner en tant qu'arbitre neutre de la réalité étatique.

\section{La relativisation de l'immunité de l'Etat en cas de graves violations des Droits de l'Homme et du Droit International humanitaire}

The law is always too short and too tight for growing bumankind. ${ }^{79}$

A supposer qu'il existe un conflit entre l'immunité de l'Etat et les règles de jus cogens, l'évolution même de la notion d'immunité dans des affaires comme la présente conduit à affirmer que la décision de la Cour est en désaccord avec le Droit International contemporain. En effet, il est essentiel de souligner la nécessité de relativiser l'immunité de l'Etat dans des situations impliquant de graves violations des Droits de l'Homme et du Droit Humanitaire, contrairement à la décision de la majorité. La relativisation de l'immunité allemande rendrait les demandes de réparation des victimes recevables auprès des tribunaux italiens. Toutefois, la Cour a estimé qu'il n'existe pas actuellement de coutume internationale qui permette d'anticiper une évolution de la pratique de l'Etat en matière d'immunité étatique, y compris en ce qui concerne de graves violations de Droits de l'Homme et du Droit Humanitaire. ${ }^{80}$

Afin de parvenir à une telle conclusion, et compte tenu du fait que les règles sur l'immunité de l'Etat sont issues du droit coutumier, la Cour a évalué l'existence d'une pratique bien établie et d'une opinio juiris, éléments constitutifs de la coutume internationale. ${ }^{81}$ Cependant, l'analyse entreprise par la Cour est critiquable lorsqu'elle met de côté l'évolution du Droit International en matière d'immunité (2.1) et lorsqu'elle considère

\footnotetext{
79 Warren, R., All the kings men, Bantam Modern Classic, 1968, p. 136, cité par Belsky, A. C., Merva, M. \& Roht-Arriaza, N., "Implied waiver under the FSIA: a proposed exception to immunity for violations of peremptory norms of international law", California Law Review, 1989, 77, (2), p. 365.

80 Cour Internationale de Justice, Immunités juridictionnelles de l'Etat (Allemagne c. Italie; Grèce intervenant), arrêt du 3 février 2012, \91.

81 Cour Internationale de Justice, Plateau continental de la mer du Nord (République Fédérale d'Allemagne c. Danemark; République Fédérale d'Allemagne c. Pays-Bas), arrêt du 20 février 1969, \77; Cour Internationale de Justice, Platean Continental (Jamabiriya arabe libyenne/Malte), arrêt du 21 mars 1984, \ 27.
} 
comme inappropriée la distinction entre actes de puissance publique et actes de gestion afin d'accorder l'immunité (2.2). Cette tendance conduit à l'érosion progressive de la souveraineté étatique en faveur des Droits de l'Homme (2.3). Renfermée dans sa logique juridique, la Cour a ignoré la reconnaissance d'une nouvelle exception à l'immunité de l'Etat en raison de la violation de règles de jus cogens (2.4).

\subsection{Le Droit International n'est pas statique: l'immunité en tant que notion relative et évolutive}

La formation, le développement, l'interprétation et l'application du Droit International ne peuvent être dissociés de la dimension intertemporelle. ${ }^{82}$ Car en effet, comme le souligne à juste titre M. le juge Cançado Trindade dans son opinion dissidente dans la présente affaire, il n'existe pas de règles immuables de Droit International. Le besoin d'étudier une situation au regard du droit qui prévalait à l'époque, était également reconnu par l'Institut de Droit International. ${ }^{83}$ Le droit et le temps, sont donc interdépendants, l'effectivité des normes de Droit International devant être étudiée en même temps que les transformations sociales. ${ }^{84}$ Comme la Cour l'a elle-même reconnu dans l'affaire Ile de Palmas de 1928, l'existence d'un droit doit être étudié en fonction de son évolution. ${ }^{85}$ Ultérieurement, dans son avis consultatif sur la Namibie de 1971, la Cour a estimé que l'interprétation d'une norme internationale doit prendre en compte les transformations temporelles et l'évolution du système juridique. ${ }^{86}$

\footnotetext{
82 Opinion dissidente de M. le Juge A. A. Cançado Trindade, Cour Internationale de Justice, Immunités juridictionnelles de l'Etat (Allemagne c. Italie; Grèce intervenant), arrêt du 3 février 2012, $\ 5$.

83 Voir Annuaire de l'Institut de Droit International, 1973 et 1975, cités par M. le Juge A. A. Cançado Trindade dans son opinion dissidente, Cour Internationale de Justice, Immunités juridictionnelles de l'Etat (Allemagne c. Italie; Grèce intervenant), arrêt du 3 février 2012, \12, p. 5.

${ }^{84}$ Opinion dissidente de M. le Juge A. A. Cançado Trindade, Cour Internationale de Justice, Immunités juridictionnelles de l'Etat (Allemagne c. Italie; Grèce intervenant), arrêt du 3 février 2012, \14.

85 Cour Permanente d'Arbitrage, affaire Ile de Palmas (Pays-Bas c. Etats-Unis), 4 avril 1928, p. 14.

86 Cour Internationale de Justice, Conséquences juridiques pour les Etats de la présence continue de l'Afrique du Sud en Namibie (Sud-ouest africain) nonobstant la résolution 276 (1970) du Conseil de Sécurité, avis consultatif du 21 juin 1971, $\ 53$.
} 
La dimension intertemporelle s'applique également à l'immunité de l'Etat. L'évolution d'une approche absolue vers une vision restrictive témoigne de l'écoulement du temps et des besoins de la société contemporaine.$^{87}$ De fait, à l'origine, le terme "immunité" 88 est apparu à la fin du XIII ${ }^{\text {ème }}$ siècle, appliqué par le roi, et ultérieurement, par la loi ${ }^{89}$ à des personnes de la noblesse ou du clergé ou à des propriétaires ou encore à des établissements ecclésiastiques. L'objectif était d'empêcher l'interférence d'un Etat, par le biais de ses tribunaux, dans les activités d'un autre Etat et de ses représentants, compte tenu de leur souveraineté (par in parem non babet imperium).$^{90}$ L'idée primordiale s'appuyait sur la courtoisie, la dignité et les bonnes relations entre Etats. ${ }^{91}$ L'immunité provient par conséquent de la souveraineté d'un Etat, et ne constitue pas un droit accordé à un Etat étranger. ${ }^{92}$ C'est à l'Etat territorial de renoncer, expressément ou implicitement, à l'exercice de sa compétence, au risque d'interférer de manière illicite dans sa souveraineté. ${ }^{93} \mathrm{Il}$ s'agit donc d'une exception à la compétence qu'un Etat exercerait normalement sur son territoire. ${ }^{94}$

Il ne s'agit pas d'une notion statique mais d'une notion qui s'adapte à l'évolution de la société contemporaine, dont la proportionnalité devra

87 Comme le souligne McGregor, L., "The depiction of immunity as static contradicts the progressively contracting coverage of immunity, precipitated by the evolution of sovereignty", op. cit., p. 913.

88 D'après G. Cornu, immunité est issue du latin immunitas, de munus "onûs" et signifie “cause d'exemption de peine" —Cornu, G. \& Association Henri Capitant (dir.), Vocabulaire juridique, 8 c̀ éd., 2007, Coll. Quadrige, PUF-.

89 Kessedjian, C., "Immunités", Répertoire Dalloz de droit international, 2012, \1, p. 5.

90 Ibid., \2, p. 5.

91 Cour Européenne des Droits de l'Homme, arrêt du 21 novembre 2001, Fogarty c. Royaume-Uni, requête $\mathrm{N}^{\circ} 37112 / 97$, $\mathbb{S} 34$. Cf. Lauterpacht, H., "The problem of jurisdictional immunities of foreign States", British Yearbook of International Law, 1951, (28), p. 221 et 230; Cassese, A., International law, $2^{\mathrm{e}}$ éd., Oxford, 2005, pp. 99-100.

92 McGregor, op. cit., p. 913.

93 Lauterpacht, op. cit., p. 229. En sens inverse, voir Dailler, Forteau \& Pellet, Droit international public, 8 è éd., LGDJ, p. 495.

94 Opinions dissidentes de M. M. les Juges Higgins, Kooijmans et Buergenthal, Cour Internationale de Justice, Mandat d'Arrêt du 11 avril 2000 (République Démocratique du Congo c. Belgique), arrêt du 14 février 2002, \$ 71. Voir également l'opinion dissidente de M. le Juge A. A. Cançado Trindade, Cour Internationale de Justice, Immunités juridictionnelles de l'Etat (Allemagne c. Italie; Grèce intervenant), arrêt du 3 février 2012, \166. 
toujours être étudiée en prenant en compte l'intérêt protégé par la norme affectée par l'immunité ainsi que les intérêts de l'Etat auquel l'immunité serait accordée. ${ }^{95}$ La règle actuelle consiste à relativiser ladite immunité comme un reflet de l'évolution des activités traditionnellement attribuées aux Etats à partir de la fin du XIX ${ }^{\mathrm{ème}}$ siècle. La pratique étatique, surtout des Cours italiennes et belges, a ancré la distinction entre les actes de puissance publiques (acta jure imperii) et les actes de gestion (acta jure gestionis ou jure privatorum), en accordant l'immunité seulement aux premiers. ${ }^{96}$ Bien que critiquée en raison de son imprécision, ${ }^{97}$ cette tendance a pris une place croissante après la Première Guerre Mondiale avec l'augmentation de la participation des Etats dans les transactions commerciales ou privées. L'objectif à l'époque n'était pas de réprimer des crimes internationaux, mais d'éviter l'incidence de l'immunité pour le cas où l'Etat agirait en tant qu'entité privée. ${ }^{98}$ En ce sens, des conventions et projets internationaux ont consacré la notion d'immunité relative. ${ }^{99}$ Ultérieurement, au début des années 80, un recul de l'intervention étatique dans l'économie a eu lieu, avec la privatisation de différentes activités auparavant considérées comme typiquement étatiques. ${ }^{100}$ Ce processus a influencé directement les immunités de l'Etat et a donné lieu à un mouvement inverse à celui identifié en raison de l'intervention de l'Etat dans le commerce privé. Cela démontre que la distinction entre des actes de puissance publique et de gestion est soumise à une interprétation évolutive et reflète les nouvelles

\footnotetext{
95 Opinions dissidentes de M. M. les Juges Higgins, Kooijmans et Buergenthal, Cour Internationale de Justice, Mandat d'Arrêt du 11 avril 2000 (République Démocratique du Congo c. Belgique), arrêt du 14 février 2002, \ 71; Kessedjian, op. cit., \8, p. 6.

96 Voir la jurisprudence belge: Rau, Vanden Abel c. Duruty, 1879, \175 et Chemin de fer Liégeois-Luxembourg c. Etat néerlandais, 1903, \301-303; et la jurisprudence italianienne: Typaldos c. Asile di Aversa, 1886, \1492-1493 et Bey di Tunisi rappresentato da Guttieres c. Elmilik, 1886, \1544-1545.

97 Il est difficile de définir les actes commis par l'Etat étranger dans le domaine privé afin de ne pas accorder d'immunité. En ce sens, voir Cassese, op. cit., p. 101.

98 Opinion dissidente de M. le Juge A. A. Cançado Trindade, Cour Internationale de Justice, Immunités juridictionnelles de l'Etat (Allemagne c. Italie; Grèce intervenant), arrêt du 3 février 2012, $\$ 168$.

99 Voir, par exemple, la Convention Européenne sur l'Immunité des Etats de 1972 et le Projet de Convention Interaméricaine sur les Immunités juridictionnelles des Etats de 1983.

100 Kessedjian, op. cit., \ 7, p. 6.
} 
priorités de la société. ${ }^{101}$ Compte tenu de cette réalité, la question s'est posée de savoir si les entités privées, qui exercent des activités typiquement étatiques, pourraient bénéficier d'immunités reconnues aux Etats dans le cas où celles-ci exerceraient de telles fonctions. ${ }^{102}$ De plus, le développement de l'arbitrage international dans les relations impliquant les Etats a contribué à l'érosion de l'immunité juridictionnelle. ${ }^{103}$

Aussi bien dans le domaine public que privé, les immunités de l'Etat deviennent toujours plus flexibles. Elles s'adaptent à l'évolution d'une société internationale dans laquelle il existe une préoccupation croissante quant à la protection des droits individuels au détriment de l'Etat, que ce soit en raison de l'implication des individus dans des transactions commerciales avec l'Etat, ou en raison de la qualité de victime de délits commis par l'Etat en question. ${ }^{104}$ En 1951, Lauterpacht affirmait qu'il existait une tendance croissante depuis la fin de la Première Guerre Mondiale à ne pas appliquer l'immunité juridictionnelle aux Etats étrangers. ${ }^{105}$ En effet, cette doctrine serait devenue obsolète et générerait potentiellement une injustice en ce qui concerne les droits individuels. Il s'agit d'une valeur relative et évolutive, dont l'importance doit être soupesée en raison de valeurs concurrentes et de leurs respectives conséquences concrètes. ${ }^{106}$ Le droit pénal international témoigne de cette évolution, qui est marquée à la fois par l'exercice de la compétence extraterritoriale de l'Etat en cas de graves crimes internationaux (compétence universelle), et par l'érosion

101 Opinions dissidentes de M. M. les Juges Higgins, Kooijmans et Buergenthal, Cour Internationale de Justice, Mandat d'Arrêt du 11 avril 2000 (République Démocratique du Congo c. Belgique), arrêt du 14 février 2002, \$ 72.

102 Kessedjian, op. cit., \ 7, p. 6.

103 Watt, H. M., "Une perspective internationaliste-privatiste”, en Verhoeven, J. (dir.), Le droit international des immunités: contestation ou consolidation?, París-Bruselas, 2004, p. 265, note 4.

104 Opinion dissidente de M. le juge Yusuf, Cour Internationale de Justice Immunités juridictionnelles de l'Etat (Allemagne c. Italie; Grèce intervenant), arrêt du 3 février 2012, \35.

105 Lauterpacht, op. cit., p. 220.

106 Bornkamm, C., "State immunity against claims arising from war crimes: the judgement of the International Court of Justice in jurisdictional immunities of the State", German Law Journal, 13, (6), pp. 773-782. De fait l'immunité de l'Etat n'est pas "gravée dans la pierre", comme le soulignent clairement M. M. les Juges Buergenthal et Kooijmans dans leur opinion dissidente dans l'affaire Mandat d'Arrêt du 11 avril 2000 (République Démocratique du Congo c. Belgique), arrêt du 14 février 2002, \ 72. 
de l'immunité des représentants étatiques lorsque des crimes internationaux sont commis. Cela vise à combattre l'impunité et à assurer une plus grande accountability. ${ }^{107} \mathrm{La}$ raison d'Etat cède la place à l'intérêt de l'humanité à s'assurer que les personnes qui commettent de graves crimes internationaux soient punies, forgeant ainsi de façon continue la pratique de l'immunité de l'Etat. ${ }^{108}$ Par conséquent, l'immunité, en tant qu'exception de Droit International coutumier, ne peut pas être traitée comme dans le passsé et se révèle totalement inadéquate à la réalité actuelle. La Cour Internationale de Justice, dans la présente affaire, est passée à côté de tels développements et n'a su donner aucun signe d'un développement progressif du Droit International. ${ }^{109}$

\subsection{L'inadéquation de la distinction entre les actes de puissance publique et actes de gestion}

Comme souligné précédemment, la distinction entre les actes de puissance publique et actes de gestion répond au besoin d'intervention de l'Etat en tant qu'entité privée. Cependant, cette distinction n'a jamais été appropriée pour répondre aux questions d'immunité. ${ }^{110}$ En effet, il est difficile de définir si les actes accomplis par l'Etat étranger s'insèrent

107 En ce sens, voir l'opinion dissidente de M. M. les Juges Higgins, Kooijmans et Buergenthal, Cour Internationale de Justice, Mandat d'Arrêt du 11 avril 2000 (République Démocratique du Congo c. Belgique), arrêt du 14 février 2002, \$ 73-74. Opinion dissidente de M. le Juge Van Den Wyngaert, Cour Internationale de Justice, Mandat d'Arrêt du 11 avril 2000 (République Démocratique du Congo c. Belgique), arrêt du 14 février 2002, \27-28 et 23. D’après le Juge, la Cour ne prend pas en compte le développement moderne du droit pénal international vers la limitation de l'immunité de l'Etat en vertu d'une plus grande accountability.

108 Opinions dissidentes de M. M. les Juges Higgins, Kooijmans et Buergenthal, Cour Internationale de Justice, Mandat d'Arrêt du 11 avril 2000 (République Démocratique du Congo c. Belgique), arrêt du 14 février 2002, \$ 75 .

109 En ce sens, voir Conforti, B., "The judgment of the International Court of Justice on the immunity of foreign States: a missed opportunity", The Italian Yearbook of International Law, 2012, XXI, (2011), pp. 132-133.

110 Lauterpacht, op. cit., pp. 224, 226-227 et 272. Comme le souligne Lauterpacht, “[...] at least in modern conditions, the distinction between acts jure gestionis and acts jure imperii cannot be placed on a sound logical basis", op. cit., p. 224. D'après M. le Juge Yusuf, "State immunity is, as a matter of fact, as full of holes as Swiss chease" —opinion dissidente de M. le juge Yusuf, Cour Internationale de Justice, Immunités juridictionnelles de l'Etat (Allemagne c. Italie; Grèce intervenant), arrêt du 3 février 2012, \26-. 
dans la sphère publique ou privée afin d'accorder ou d'écarter l'immunité. Actuellement, deux critères sont fréquemment utilisés pour évaluer si un acte relève du jure imperii: la nature de l'acte en question et la fonction que l'acte exerce. ${ }^{111}$ D'après M. Weiss, ancien juge de la Cour Permanente de Justice Internationale dans son cours présenté à l'Académie de Droit International de La Haye, le critère de la nature de l'acte serait plus adapté, car il serait possible d'évaluer si un acte est typiquement étatique ou s'il pourrait être accompli dans les mêmes conditions par un individu. ${ }^{112}$ Cependant, une telle distinction, comme démontré ci-dessus, n'est plus conforme à l'évolution des fonctions actuellement attribuées aux Etats. Dans la présente affaire, des massacres de civils et la déportation de civils et militaires pour les soumettre aux travaux forcés en Allemagne et dans les terrritoires occupés ont eu lieu. De tels actes constituent de graves violations du Droit International Humanitaire, ayant la répercussion de crime international. Autant la nature de l'acte — crimes internationaux- que la fonction ou l'objectif qu'il est envisagé d'atteindre ne constituent pas des critères propres à le qualifier comme étant typiquement étatique, et donc passible d'immunité. Dans certaines situations, les critères portant sur la nature et fonction de l'acte peuvent aboutir à des résultats divergents, et peuvent être traités différemment en fonction du tribunal saisi. ${ }^{113} \mathrm{La}$ pratique de l'Etat au travers de diverses décisions judiciaires corrobore cet argument. ${ }^{114}$

111 Cassese, A., International law, $2^{\grave{c} e ́ d ., ~ O x f o r d, ~ 2005, ~ p . ~} 101$.
112 Weiss, A., “Compétence ou incompétence des tribunaux à l'égard des Etats étrangers",
RCADI, 1923, 1, pp. 521-552.
113 En ce sens, Antônio Cassese donne un exemple en citant le cas d'un Etat étranger qui
achète des biens pour ses forces armées. Quant à leur nature —l'achat de biens- l'acte
serait considéré comme privé par nature et, par conséquent, il n'y aurait pas d'immunité.
Quant à la fonction qu'il poursuit —achat de biens pour les forces armées, l'acte est typi-
quement étatique et, par conséquent il y aurait immunité- (Cassese, op. cit., p. 101). Pour
plus de détails concernant la pratique de différents tribunaux étatiques qui ont interprété
différemment la distinction entre les actes de puissance publique et de gestion, voir Lauter-
pacht, op. cit., pp. 222-223.
114 Voir, par exemple, l'arrêt de la Cour Suprême d'Israel dans l'affaire Eichmann; Cour
Suprême, 29 mai 1962, International Law Reports, 36, p. 312; discours des Lords Hutton et
Phillips of Worth Matravers dans R. v. Bartle and the Con₹missioner of Police for the Metropo-
lis und Otlzers, ex partie Pinochet ('Pinochet III'); et discours de Lords Steyn et Nicholls of
Birkenhead dans 'Pinochet I'; arrêt de la Cour d'Appel d'Amsterdam dans l'affaire Bouterse
(Gerechtshof Amsterdam, 20 novembre 2000, $\int 4.2$ ), cités dans l'opinion dissidente de 
L'inadéquation desdits critères mène à se demander si des atrocités militaires, commises en accord avec la politique d'Etat, entreraient dans la catégorie des actes de puissance publique, c'est-à-dire qu'elles seraient qualifiées de fonctions typiquement étatiques. Constitueraient-elles des actes de souveraineté de nature à empêcher la mise en cause de la responsabilité étatique auprès des tribunaux civils de ses pairs ? ${ }^{115}$ Evidemment, des violations de normes impératives ne pourraient pas être intégrées dans la catégorie des actes de puissance publique, même si celles-ci étaient commises par un Etat. ${ }^{116}$ En effet, la pratique de crimes internationaux n'est pas une fonction typiquement étatique ou bien que seul un Etat pourrait exercer. ${ }^{117} \mathrm{Il}$ est clair que des crimes internationaux ne sont pas des actes de gestion, ni des actes de puissance publique. ${ }^{118} \mathrm{Il}$ s'agit plutôt d'actes contraires au Droit International, qui à eux-seuls écarteraient toute allégation d'immunité, ce qui semble une tendance doctrinale. ${ }^{119} \mathrm{D}$ 'après le juge dissident Antônio Augusto Cançado Trindade, "un crime est un crime, indépendamment de celui qui l'a commis". ${ }^{120}$ En l'espèce, il s'agit

M. M. les Juges Higgins, Kooijmans et Buergenthal, Cour Internationale de Justice, Mandat d'Arrêt du 11 avril 2000 (République Démocratique du Congo c. Belgique), arrêt du 14 février 2002, p. 3, $\int 85$.

115 Watt, H. M., "Les droits fondamentaux devant les juges nationaux à l'épreuve des immunités juridictionnelles: à propos de l'arrêt de la Cour Internationale de Justice, immunites juridictionnelles de l'Etat (Allemagne c. Italie; Grèce intervenant), du 3 février 2012", p. 5.

116 La catégorie des actes jure imperii ne peut pas couvrir des violations de normes impératives (Watt, op. cit., pp. 10-11).

117 Opinion dissidente de M. M. les Juges Higgins, Kooijmans et Buergenthal, Cour Internationale de Justice, Mandat d'Arrêt du 11 avril 2000 (République Démocratique du Congo c. Belgique), arrêt du 14 février 2002, p. 3, \85.

118 Voir l'opinion dissidente de M. le Juge A. A. Cançado Trindade, Cour Internationale de Justice, Immunités juridictionnelles de l'Etat (Allemagne c. Italie; Grèce intervenant), arrêt du 3 février 2012, \177, p. 52.

119 Trapp, K. N., "Smooth runs the water where the brook is deep: the obscured complexities of Germany v. Italy", Cambridge Journal of International and Comparative Law, 2002, 1, (1), pp. 153-168; Bianchi, A., "Denying State immunity to violators of human rights", Austrian Journal of Public and International Law, 1994, 46, pp. 227-228; Orakhelashvili, "State immunity and hierarchy of norms...", op. cit., p. 970.

120 Opinion dissidente de M. le Juge A. A. Cançado Trindade, Cour Internationale de Justice, Immunités juridictionnelles de l'Etat (Allemagne c. Italie; Grèce intervenant), arrêt du 3 février 2012, \184, p. 53. 
de delicta imperii, à savoir un crime international commis en violation d'une norme impérative, à l'encontre duquel il ne serait pas possible d'invoquer d'immunité. ${ }^{121} \mathrm{Il}$ est donc évident que la distinction entre jure imperii et jure gestionis est clairement inadéquate à l'évolution du Droit International, et surtout dans la présente affaire.

\subsection{L'érosion progressive de la souveraineté étatique en faveur des droits individuels}

Comme cela a été vu précédemment, la notion d'immunité d'État provient historiquement de la dignité des Etats, qui a survécu à une époque à laquelle le souverain, en tant que legibus solutus, ne rendait la justice aux sujets qu'en vertu de la grâce et non du respect de la règle de droit. ${ }^{122}$ L'évolution actuelle du Droit International est marquée par la reconnaissance des individus en tant que sujets de droits et devoirs qui émanent du Droit International. En témoigne l'évolution du Droit International des Droits de l'Homme et du Droit International Humanitaire, du Droit International des Réfugiés et du Droit Pénal International. ${ }^{123} \mathrm{Il}$ ne fait aucun doute que de telles considérations doivent être prises en compte dans la pratique actuelle liée à l'immunité de l'Etat, ce qui conduit à sa limitation progressive. D'après Lauterpacht, la flexibilisation de l'immunité de l'Etat est essentielle en raison de l'expansion des activités des Etats et de l'injustice qui résulte du rejet des demandes des individus. ${ }^{124}$

\footnotetext{
121 Idem.

122 Lauterpacht H., op. cit., p. 226. Voir l'opinion dissidente de M. le Juge A. A. Cançado Trindade, Cour Internationale de Justice, Immunités juridictionnelles de l'Etat (Allemagne c. Italie; Grèce intervenant), arrêt du 3 février 2012, \176, p. 51.

123 Voir l'opinion dissidente de M. le Juge A. A. Cançado Trindade, Cour Internationale de Justice, Immunités juridictionnelles de l'Etat (Allemagne c. Italie; Grèce intervenant), arrêt du 3 février 2012, \176, p. 51.

124 D'après Lauterpacht, "in so far as the jurisdictional immunity of foreign states is assumed to be based on their dignity, the time has probably come for abandoning what is now no more than an incantation alien to the conception of the rule of law, national and international, and to the true position of the State in modern society" (ibid., p. 232). Il poursuit: "At a period in which in enlightened communities the securing of the rights of the individual, in all their aspects, against the state has become a matter of special and significant effort, there is no longer a disposition to tolerate the injustice which may arise whenever the state — our own state or a foreign state — screens itself behind the shield of immunity in order to defeat a legitimate claim” (ibid., p. 235).
} 
On peut donc constater que l'immunité de l'Etat n'est pas une notion statique. Au contraire, elle est actuellement restreinte en faveur de la reconnaissance des droits individuels tant dans le domaine commercial que pénal. Il en découle que la même idée s'applique lorsqu'ont lieu de graves violations de Droits de l'Homme et du Droit International Humanitaire. S'il y a une immunité de l'Etat pour des questions commerciales, pourquoi n'y en aurait-il pas pour des situations encore plus graves qui impliquent des violations de normes péremptoires du Droit International ? Etant donné que l'immunité de l'Etat relève actuellement du Droit International coutumier, rien n'empêcherait de reconnaitre une évolution continue du Droit International tendant à faire progresser la pratique de l'immunité de façon à répondre aux besoins de la société contemporaine. ${ }^{125}$ Le fondement même de l'immunité de l'Etat est contestable. Alors que certains défendent que l'immunité a pour origine la dignité et courtoisie entre les Etats, étant une exception à la compétence juridictionnelle qu'un Etat exercerait normalement sur son territoire et non pas un droit d'un Etat étranger, ${ }^{126}$ d'autres défendent que l'immunité est issue de l'égalité souveraine des Etats, dont l'objectif est d'assurer le respect de la souveraineté de l'Etat lorsque ses agents, sa législation ou ses biens se rattachent à la souveraineté territoriale d'un autre Etat. ${ }^{127}$ D'après ce dernier courant, le fait d'écarter l'immunité entraînerait la violation de l'égalité souveraine. Indépendamment de ce fait, il est incontestable que l'idée de souveraineté a évolué au cours des temps.

De fait, après les crises humanitaires survenues au cours de la décennie de 1990 surtout au Rwanda et dans les Balkans, il a été nécessaire de redéfinir la souveraineté de l'Etat. La souveraineté implique d'assurer la protection de la population (sovereignty as responsibility) et d'agir de façon préventive. La communauté internationale ne saurait se dérober face à de graves abus de Droits de l'Homme commis par des Etats souverains à l'encontre de ses propres citoyens. En effet, la souveraineté individuelle,

\footnotetext{
125 Opinion dissidente de M. le Juge Koroma, Cour Internationale de Justice, Immunités juridictionnelles de l'Etat (Allemagne c. Italie; Grèce intervenant), arrêt du 3 février 2012: "Nothing in the court's judgement today prevents the continued evolution of law on State immunity" ( $(\mathbb{3} 7)$.

126 Lauterpacht, H., "The problem of jurisdictional immunities of foreign States", British Yearbook of International Law, 1951, (28).

127 Daillier, P., Forteau, M. \& Pellet, A., Droit international public, $8^{e ̀ ~ e ́ d ., ~ L G D J, ~ P a r i ́ s, ~}$ 2009, p. 497.
} 
à savoir, la liberté fondamentale de chaque individu, est progressivement mise en valeur compte tenu de la prise de conscience croissante des droits individuels. Ainsi, si l'Etat ne protège pas sa population, la responsabilité de la communauté internationale se superpose à la non-intervention. D'après Kofi Annan, il est essentiel que la communauté internationale parvienne à un consensus selon lequel des violations massives et systématiques des Droits de l'Homme ne peuvent pas rester impunies. A partir d'un tel constat, il y a eu une acceptation progressive du principe de la Reponsabilité de Protéger de la part de la communauté internationale, qui a remplacé la notion largement controversée d' "intervention humanitaire". ${ }^{128}$ Les termes mêmes du débat ont été modifiés, de 'souveraineté en tant que contrôle', à 'souveraineté en tant que responsabilité'. ${ }^{129}$

Actuellement, il est admis que la souveraineté étatique doit être au service des individus. Un Etat ne saurait s'abriter derrière la souveraineté afin de commettre ou laisser se commettre de graves violations des Droit de l'Homme et du Droit International Humanitaire. L'érosion progressive de la souveraineté étatique en faveur des droits individuels est un phénomène qui se constate dans différents domaines du Droit International, étant également applicable à la présente affaire. Il peut donc être vérifié que l'immunité de l'Etat est actuellement restreinte bien que fondée sur l'idée de souveraineté.

\footnotetext{
128 Fondé sur l'idée selon laquelle la souveraineté est responsabilité, le principe de Responsabilité de Protéger a été présenté pour la première fois dans le rapport de la Commission Internationale sur l'Intervention et la Souveraineté de l'Etat (Iciss) en décembre 2001. Il a ultérieurement été promu par le Secrétaire Général Kofi Annan em septembre 2003 et inclus dans le rapport du groupe de haut niveau "Un monde plus sûr: notre affaire à tous" émis en décembre 2004. Ce document a servi de base au propre rapport du Secrétaire Général intitulé "Dans une liberté plus grande - Vers le développement, la sécurité et les droits de l'homme pour tous", qui a souligné la responsabilité des gouvernements dans la protection de leurs propres peuples, sachant que la responsabilité ne retombe sur la communauté internationale que lorsque l'Etat n'est pas capable ou n'agit pas afin de protéger ses citoyens (l'usage de la force étant toujours en dernier recours). Cependant, l'engagement historique dans le cadre de l'onU vient de l'adoption du Document Final du Sommet Mondial en septembre 2005 (voir $\$ 138-139$ ).

129 Annan, K., "Two concepts of sovereignty", The Economist, September $18^{\text {th }} 1999$; voir Peters, A., "Le droit d'ingérence et le devoir d'ingérence: vers une responsabilité de protéger", Revue de Droit International et de Droit Comparé, 2002, 79, pp. 290-308; Peters, A., "The security council's responsibility to protect", International Organizations Law Review, 2011, 8, p. 5.
} 


\subsection{L'apparition d'une nouvelle exception à l'immunité de l'Etat en raison de violation de règles de jus cogens}

La possibilité de relativiser l'immunité d'Etat en raison de violation de règles de jus cogens n'a pas encore été reconnue par le droit positif international, à savoir par la Convention Européenne sur l'Immunité des Etats de 1972, adoptée dans le cadre du Conseil de l'Europe (Convention de Bâle) et par la Convention des Nations Unies sur les Immunités Juridictionnelles des Etats et de leurs Biens de 2005. Toutefois, cela n'empêche pas de reconnaître que le Droit International a progressivement évolué en la matière. La Commission du Droit International, chargée de préparer la future convention dans le cadre de l'ONU, a démarré ses travaux en 1979 et les a conclus en 1991 avec l'adoption d'un projet d'articles sur les immunités juridictionnelles des Etats et de leurs biens. Le projet a seulement été transformé en convention près de treize ans plus tard. Cependant, ladite convention n'est pas encore entrée en vigueur car elle n'a pas obtenu les trente ratifications requises par l'article $30 .{ }^{130}$ Le texte définitif, éminemment conservateur, ne prévoit aucune disposition restreignant l'immunité de l'Etat en cas de violation de règles de jus cogens. ${ }^{131}$ Malgré cela, les membres du groupe de travail de la Commission du Droit International n'ont pas manqué de souligner les développements récents de la pratique étatique et de la législation en matière d'immunité dans des affaires impliquant des violations de règles de jus cogens. Dans le rapport, ils ont cité des demandes qui visaient à écarter l'immunité en raison de graves violations des Droits de l'Homme, notamment dans des situations relevant de l'interdiction de

130 Actuellement, la Convention compte 28 Etats signataires et 14 Etats-membres. Disponible sur: http://treaties.un.org/pages/ViewDetails.aspx?src=TREATY\&mtdsg_ no $=$ III-13\&chapter $=3 \&$ lang $=$ en

131 Le commentaire de la Commission de Droit International au projet de l'article 12, relatif aux dommages causés à des personnes et biens - ladite tort exception, souligne que cet article ne s'applique pas à des situations impliquant des conflits armés (Annuaire de la Commission de Droit International, Vol. II, 1991, parte II, p. 46). D'après Gerhard Hafner, président du comité ad hoc, qui a recommandé l'adoption de la Convention, il y avait un consensus général selon lequel des situations impliquant des conflits armés ne seraient pas couvertes par la Convention. Au moment de la ratification, l'Italie, la Norvège et la Suède ont émis des déclarations clarifiant que la convention ne s'appliquerait pas aux activités armées ou militaires. Pour plus de détails, voir Opinion dissidente de M. le Juge ad hoc Gaja, Cour Internationale de Justice, Immunités juridictionnelles de l'Etat (Allemagne c. Italie; Grèce intervenant), arrêt du 3 février 2012, \$6. 
la torture. La Commission a toutefois reconnu que si certains tribunaux ont fait preuve de sympathie pour cet argument, l'immunité de l'Etat a prévalu dans la plupart des cas. Elle cite l'amendement au Foreign Sovereign Immunities Act (FSLA) et l'affaire Pinochet comme témoins de la flexibilisation de l'immunité d'Etat. ${ }^{132}$ Elle en déduit que les développements relatifs à l'opposition entre l'immunité et les règles de jus cogens, bien que n'étant pas prévus dans le projet d'articles de la convention, constituent des développement récents relatifs à l'immunité qui ne sauraient être ignorés. ${ }^{133}$

De fait, les juges nord-américains ont appliqué l'exception à l'immunité de l'Etat prévue dans le FSLA dans différents jugements mettant en cause le terrorisme et les Droits de l'Homme. ${ }^{134}$ Le FSLA a donc été modifié afin d'inclure une nouvelle exception à l'immunité de l'Etat en cas d'attaques terroristes. Une telle exception était prévue dans la section 221 de l'Anti Terrorism and Effective Death Penalty Act de 1996. Ce dernier prévoyait que l'immunité ne serait pas accordée en cas de demandes en réparation de dommages à l'encontre d'un Etat étranger pour des préjudices personnels ou mort causée par des actes de torture, de meurtre extrajudiciaire, de sabotage d'aéronefs, de prise d'otages. La terrorism exception prévue dans le FSLA permet aux victimes américaines d'engager des procès non seule-

132 L'affaire Pinochet, citée de façon exhaustive par la doctrine et soulignée dans le rapport du groupe de travail de la Commission de Droit International sur les immunités juridictionnelles des Etats et de leur propriété, a également insisté sur les limites de l'immunité de l'Etat dans des situations dans lesquelles ont lieu de graves violations des droits de l'homme commises pas des représentants d'Etats — Rapport de la Commission de Droit International (1999), A /54/10, PJ (rapport du groupe de travail sur les immunités juridictionnelles des Etats et leurs biens), Commission de Droit International, 1999, II, (2), p. 172, \13-. La 'House of Lords' avait décidé qu'un ancien chef d'Etat ne pourrait pas invoquer l'immunité en ce qui concerne des actes de torture, qui feraient partie intégrante des interdictions de jus cogens, commis dans son propre Etat. Un tel arrêt permettrait d'entrevoir l'évolution dans le domaine de l'immunité en raison d'actes de torture autant dans des procès civils que dans des procès pénaux — Rapport de la Commission de Droit International (1999), A/54/10, PJ (rapport du groupe de travail sur les immunités juridictionnelles des Etats et de leur propriété), Commission de Droit International, 1999, II, (2), p. 172, \12-. Pour plus de détails, voir Stern, B., "Can a State or a head of State claim the benefit of immunities in case an international crime has been committed?", ILSA Journal of International and Comparative Law, 2008, 14, (2).

133 Rapport de la Commission du Droit International (1999), A/54/10, PJ (rapport du groupe de travail sur les immunités juridictionnelles des Etats et de leurs biens), Commission de Droit International, 1999, II, (2), p. 172, \$13.

134 Foreign Sovereign Immunities Act, section 1605 (a)(7). 
ment à l'encontre d'Etats étrangers, mais aussi à l'encontre de ses agents, désignés par les USA comme ayant promu le terrorisme. En raison des difficultés rencontrées par les victimes pour faire valoir leurs droits contre les Etats étrangers sur la base du FSIA, une nouvelle loi a été approuvée en 2008 (National Defense Authorization Act for Fiscal Year 2008 - NDAA). Celle-ci prévoit le droit d'agir contre des Etats considérés comme ayant promu le terrorisme, et permet la condamnation à des peines comminatoires. ${ }^{135} \mathrm{La}$ flexibilisation de l'immunité de l'Etat a été appliquée à différentes affaires, impliquant surtout la Lybie, l'Iran, Cuba, le Soudan et la Corée du Nord. ${ }^{136}$ Il convient de mentionner également l'affaire Samantar c. Yousuf relative à des actes de torture pratiqués en Somalie. ${ }^{137}$ Cette affaire a fait l'objet d'un recours à la Cour Suprême des USA en juin 2010, dans lequel il a été considéré que le FSLA ne s'appliquerait pas à une éventuelle immunité de fonctionnaires étrangers. ${ }^{138} \mathrm{La}$ décision renforce la tendance à s'écarter de l'immunité de l'Etat en cas de violations de règles péremptoires du Droit International, ce qui a été corroboré par différents amicus curiae soumis à la Cour Suprême. ${ }^{139}$

L'évolution de la pratique nord-américaine tendant à repousser l'immunité de l'Etat a largement été discutée par la doctrine. ${ }^{140}$ D'après les

135 La section 1083 du NDAA a substitué la section 1605 (a)(7) avec une nouvelle exception liée au terrorisme, qui a été codifiée comme 1605A. Plus de 35\% des décisions impliquant le FSIA en 2008 étaient liées à des demandes terroristes, avec une augmentation de plus de $15 \%$ par rapport à 2002 et $25 \%$ par rapport à 1998 (Crowell et Moring, The Foreing Sovereign Immunities Act, 2008 Year in Review, disponible sur www.crowell.com).

136 Pour une liste des décisions relatives à des demandes terroristes dans le cadre du FSIA, voir Crowell et Moring, The Foreing Sovereign Immunities Act, 2008 Year in Review, pp. 20-21, note 96.

137 Dans cette affaire, cinq Somaliens demandaient réparation à Mohamed Ali Samantar, ex-ministre de la Défense et Premier ministre de la République Démocratique de Somalie. Il soutenait que Samantar aurait été responsable d'actes de torture, viol, prison arbitraire et exécution extrajudiciaire qui ont été infligés à des individus et à leurs familles en Somalie dans les années 1980.

138 Cour Suprême des Etats-Unis, $1^{\text {er }}$ juin 2010, Samantar c. Yousuf, 660 us 2010.

139 Pour plus de détails, voir Knuchel, S., "State immunity and the promise of jus cogens", Northwestern Journal of International Human Rights, 2011, 9, (2), p. 149, note 4.

140 En ce sens, voir Knuchel, op. cit., pp. 149-183; Belsky, A. C., Merva, M. \& Roht-Arriaza, N., "Implied waiver under the FSIA: a proposed exception to immunity for violations of peremptory norms of international law", California Law Review, 1989, 77, (2), pp. 365-415; Johnson, T. A., "A violation of jus cogens norms as an implicit waiver of immunity under 
dispositions du FSLA, la violation de règles de jus cogens constitue une renonciation implicite —implied waiver —à l'immunité étatique. ${ }^{141}$ Cependant, la plupart des récents développements de la jurisprudence nord-américaine en ce qui concerne l'immunité de l'Etat n'a pas été pris en compte par la CIJ dans l'affaire Allemagne c. Italie, alors que d'autres ont été évalués de façon sélective et contestable. D'après Riccardo Pavoni, le choix des affaires citées par l'arrêt de la Cour démontre une position conservatrice et finaliste qui a pour objectif de soutenir une position antérieure qui avait déjà été prise par la majorité des juges. ${ }^{142}$ Selon le même auteur, l'arrêt de la CIJ dans la présente affaire ne reflète pas le Droit International contemporain en matière d'immunité d'Etat en raison de graves violations de Droits de l'Homme. ${ }^{143}$

La tendance à limiter l'immunité de l'Etat mise en valeur par Lauterpacht au début des années 50 et soulignée par la Commission du Droit International en 1999, suit son cours dans d'autres pays. En ce sens, il est possible de citer l'affaire Ferrini auprès de la Cour de Cassation italienne de 2004, qui a donné lieu à la demande auprès de la CIJ, ${ }^{144}$ ainsi que l'affaire Milde c. Civitella, impliquant le massacre dans la ville italienne de

the Federal Sovereign Immunities Act”, Maryland Journal of International Law, 1995, 19, (2), pp. 259-291.

141 Dans l'affaire Princz. c. République Fédérale d'Allemagne, alors que la Cour municipale nord-américaine a écarté l'immunité allemande et a reconnu le droit à réparation d'un juif nord-américain qui avait été soumis à des traitements dégradants en camp de concentration, la Cour d'Appel nord-américaine a cassé cette décision, en accordant l'immunité à l'Etat en cause. La Juge Patrícia Wald dans son opinion dissidente a souligné la gravité des actes commis à l'encontre du demandeur, qui constituaient des violations de normes de jus cogens, qui écarteraient à elles seules l'immunité de l'Etat. D'après la juge, cette interprétation est conforme au Foreign Sovereign Immunities Act - FSLA (art. 612-19). Pour plus de détails, voir Johnson, ibid., p. 259 et suiv.

142 Pour une analyse des ommissions et évaluations incomplètes effectuées par la CIJ en ce qui concerne la jurisprudence nord-américaine, voir Pavoni, R., "An American anomaly? On the ICJ's selective reading of United States practice in jurisdictional immunities of the State", The Italian Yearbook of International Law, 2012, XXI, (2011), pp. 143-159.

143 Ibid., p. 144.

144 Ferrini c. République Fédérale d'Allemagne, décision No 5044/2004. Pour des commentaires, voir Bianchi, A., "Ferrini v. Federal Republic of Germany", The American Journal of International Law, 2005, 99, (1), pp. 242-248; De Sena, P. \& De Vittor, F., "State immunity and human rights: the Italian Supreme Court decision on the Ferrini case", The European Journal of International Law, 2005, 16, (1), pp. 89-112. 
Civitella, également jugée par ladite Cour de Cassation en 2008 et 2009. Il s'agissait d'un procès pénal à l'encontre d'un officier nazi ayant participé au massacre qui a eu lieu en 1944. Dans sa décision de 2009, la Cour de Cassation a confirmé la position selon laquelle l'immunité de l'Etat ne serait pas applicable en cas de crimes internationaux qui constituent des violations de jus cogens, et a dédommagé les victimes et leurs parents de la part de l'Allemagne et de Middle. Comme le souligne le juge Antônio Cançado Trindade dans son opinion dissidente, le key-point de la décision de la Cour de Cassation italienne consistait à ne pas accorder d'immunité à l'Etat lorsque la politique pénale poursuivie par celui-ci menait à la commission de crimes contre l'humanité. ${ }^{145}$

La jurisprudence grecque dans l'affaire Distomo a suivi la même ligne jusqu'à la décision dans l'affaire Margellos. Le massacre de Distomo se rapporte à l'extermination nazie de près de 218 personnes dans le village grec de Distomo, près de Delfos, en 1944. ${ }^{146}$ La demande civile de parents de victimes à l'encontre de l'Allemagne a été soumise à la Cour de Première Instance de Livadie en 1995. Celle-ci a établi la responsabilité de l'Allemagne et a accordé une indemnisation de trente millions aux victimes du massacre de Distomo. Tant la Cour du District en 2007 que la Cour de Cassation grecque, dans l'affaire Areios Pagos jugée en 2000, ont estimé que l'Allemagne ne pourrait pas se prévaloir de l'immunité juridictionnelle, étant donné que cette immunité n'est pas absolue y compris en ce qui concerne des actes accomplis jure imperii. La Cour de Cassation a estimé qu'il y avait renonciation tacite à l'immunité de l'Etat à chaque fois que les actes en question constituaient des violations de règles de $j u s$ cogens. Ainsi, la décision de la Cour de Première Instance accordant une indemnisation est devenue définitive. En raison du non-respect de cette décision par l'Allemagne, les demandeurs ont cherché à obtenir l'exécution forcée par le biais du blocage de propriétés allemandes en Grèce. A cet effet, il serait nécessaire d'obtenir le consentement du ministre de la Justice comme prévu à l'article 923 du Code de Procédure Civile Grec, ce

\footnotetext{
145 Opinion dissidente de M. le Juge A. A. Cançado Trindade, Cour Internationale de Justice, Immunitésjuridictionnelles de l'Etat (Allemagne c. Italie; Grèce intervenant), arrêt du 3 février 2012, \191.

146 Pour plus de détails concernant chacune des affaires en question, voir l'opinion dissidente de M. le Juge A. A. Cançado Trindade, Cour Internationale de Justice, Immunités juridictionnelles de l'Etat (Allemagne c. Italie; Grèce intervenant), décision du 4 juillet 2011 (requête de la République Hellénique demandant la permission d'intervenir), \$ 29.
} 
qui n'a pas été accordé en l'espèce. Par conséquent, les demandeurs ont saisi la CEDH sur la base de l'article 6 (1) de la Convention Européenne des Droits de l'Homme. La Cour a toutefois déclaré la demande irrecevable. ${ }^{147}$ En parallèle, une affaire similaire était en cours de jugement auprès des Cours grecques. Il s'agit de l'affaire Margellos et autres, devant la Cour Suprême Spéciale grecque. A la majorité de six votes contre cinq, elle a décidé, au regard du Droit International coutumier, qu'un Etat étranger dispose d'une immunité souveraine en cas de dommages causés sur l'Etat du territoire, ceci indépendamment du fait qu'une telle conduite ait violé des règles de jus cogens ou que les forces armées aient participé au conflit armé. A l'inverse, cinq juges ont affirmé dans leurs opinions dissidentes que l'interdiction de crimes de guerre avait le statut de norme péremptoire de Droit International. Par conséquent, la décision de l'affaire Margellos en 2002 a mené à la cassation de la décision de la Cour de Première Instance de Livadie qui avait accordé une indemnisation aux demandeurs. ${ }^{148}$

Récemment, en 2011, la Cour de Cassation française a reconnu que la violation d'une règle de jus cogens "peut constituer une restriction légitime à l'immunité juridictionnelle". ${ }^{49} \mathrm{Il}$ s'agit d'un véritable revirement de jurisprudence, car dans des affaires précédentes la même Cour avait refusé de se prononcer sur la possibilité de payer des salaires à des individus soumis aux travaux forcés en Allemagne pendant la Seconde Guerre Mondiale. ${ }^{150} \mathrm{Il}$ est donc possible que la Cour analyse des procès civils pour violation de règles de jus cogens, écartant de ce fait l'immunité en tant que pouvoir souverain de l'Etat. ${ }^{151}$ Ladite décision, tout comme

\footnotetext{
147 Cour Européenne des Droits de l'Homme, arrêt du 12 décembre 2002, Kalogeropoulou et autres c. Grèce et Allemagne, requête No 59021/00.

148 Pour plus de détails concernant chacune des affaires en question, voir l'opinion dissidente de M. le Juge A. A. Cançado Trindade, Cour Internationale de Justice, Immunités juridictionnelles de l'Etat (Allemagne c. Italie; Grèce intervenant), décision du 4 juillet 2011 (requête de la République Hellénique demandant la permission d'intervenir), \11-20.

149 Civ. $1^{\text {ère }}, 9$ de mars 2011, No 09-14.743.

150 Civ. $1^{\text {ìre }}, 16$ décembre 2003, Nº 01-45.961 et 3 janvier 2006, $N^{\circ}$ 04-47.504. Ces décisions étaient liées à la déportation et à la soumission de citoyens français aux travaux forcés en Allemagne. La Cour de Cassation avait considéré que l'acte avait la nature de jure imperii.

151 Dans l'affaire mentionnée, la Cour a estimé que l'interdiction d'actes terroristes, qualifiée de règle de jus cogens, bien que constituant une restriction légitime à l'immunité de juridiction, serait disproportionnée compte tenu de l'objectif poursuivi dans le cas d'espèce. Voir pour plus de détails, Kessedjian, op. cit., p. 27.
} 
celle de la Cour Supérieure du Québec ont été citées par l'Italie au cours du procès de manière à renforcer la restriction progressive de l'immunité en ce qui concerne des actes jure imperii. ${ }^{152}$

Plusieurs affaires ont été jugées par la CEDH concernant la relation entre l'immunité de l'Etat et les règles de jus cogens, comme celles $\mathrm{Al}$ Adsani c. Royaume-Uni, ${ }^{153}$ McElhinney c. Irlande, ${ }^{154}$ et Kalogeropoulou et autres c. Grèce et Allemagne. ${ }^{155}$ Dans l'affaire Al-Adsani, largement débattue par la doctrine, la Cour a estimé qu'aucun des accords internationaux qui traite de l'interdiction de la torture en tant que norme impérative du Droit International ne se rapporte à une procédure civile ou à une immunité de l'Etat. Or de tels accords ont été adoptés avant l'admission de la thèse restreinte en matière d'immunité d'Etat et avant l'évolution des procès civils en raison de la violation des Droits de l'Homme. La décision a été prise à une faible majorité (9 votes contre 8 ), l'opinion dissidente des juges Rozakis et Caflisch ayant été suivie par 4 autres juges. Cette dernière a souligné qu'un Etat ne peut pas s'abriter derrière des règles sur l'immunité de l'Etat afin d'éviter les conséquences de ses actions, et surtout afin d'éviter des procès civils en réparation de dommages issus d'actes de torture auprès d'une juridiction étrangère. ${ }^{156} \mathrm{La}$ Cour elle-même, par décision, a reconnu le caractère transitoire des règles coutumières concernant l'immunité de l'Etat et la possibilité de la limiter, laissant la porte ouverte aux futurs développements de la matière dans la prolongement des opinions dissidentes. ${ }^{157}$

Dans l'affaire McElhinney c. Irlande, la CEDH suit le même chemin. Elle a reconnu dans son arrêt du 21.11.2001, que bien qu'il existe une tendance

152 Cour Suprême du Québec, décision du 25 janvier 2011, Kaz̨emi (Estat de) et Hashemi c. Iran, Ayatollah Ali Khamenei et autres, 2011, QCCs 196.

153 Cour Européenne des Droits de l'Homme, arrêt du 21 novembre 2001, Al-Adsani c. Royaume-Uni, requête $\mathrm{N}^{\circ} 35763 / 97$.

154 Cour Européenne des Droits de l'Homme, arrêt du 21 novembre 2001, McElbinney c. Irlande, requête $\mathrm{N}^{\circ} 31253 / 96$.

155 Cour Européenne des Droits de l'Homme, arrêt du 12 décembre 2002, Kalogeropoulou et autres c. Grèce et Allemagne, requête $\mathrm{N}^{\circ}$ 59021/00.

156 Opinions dissidentes de M. M. les Juges Rozakis, Caflish, Wildhaber, Costa, Cabral Barreto et Vajic, arrêt du 21 novembre 2001, Al-Adsani c. Royaume-Uni, requête $\mathrm{N}^{\circ} 35763 / 97, \S 3$.

157 Rozakis, C. L., "The law of State immunity revisited: the case law of the European Court of Human Rights”, Revue bellénique de droit international, 2008, 61, p. 593. 
en Droit International et comparé vers la limitation de l'immunité de l'Etat pour des dommages causés par une action ou une ommission commise sur le territoire de celui-ci, cette pratique de l'Etat ne serait pas universelle. Les juges dissidents Rozakis et Loucaides ont souligné que la Cour n'a pas pris en considération les développements du Droit International, ayant restreint de façon disproportionnée le droit d'accès aux tribunaux. De fait il existe une tendance selon laquelle la doctrine de l'immunité de l'Etat fait l'objet de plus en plus d'exceptions, parmi lesquelles la protection des Droits de l'Homme. ${ }^{158}$ Comme le juge Loucaides le souligne, dans la société démocratique actuelle, l'immunité absolue semble être une doctrine anachronique incompatible avec les demandes en justice et la règle de droit. ${ }^{159}$ D'autres juges dissidents ont également mis en évidence la tendance de restriction de l'immunité de l'Etat surtout au début du XX $\mathrm{X}^{\text {ème }}$ siècle, lorsque l'Etat participe aux activités commerciales. ${ }^{160}$

Dans la même ligne, l'affaire Kalogeropoulou et autres c. Grèce et Allemagne, la CEDH a reconnu que le droit d'accès à la justice pourrait faire l'objet d'une limitation, dès lors que de telles limitations ne portent pas atteinte à l'essence même de ce droit, et poursuivent un but légitime et proportionnel. ${ }^{161} \mathrm{La}$ Cour a estimé qu'il était inacceptable que des Etats ne bénéficient pas d'immunité pour des demandes civiles en réparation devant le tribunal d'un autre Etat en raison des crimes contre l'humanité - un tel constat cependant n'empêche pas le futur développement du droit coutumier international. Alors que la Cour reconnait l'évolution en cours du Droit International, les opinions dissidentes des affaires rapportées cidessus défendent une solution plus progressiste, permettant de prendre en compte la protection des Droits de l'Homme. Ainsi, il existe une tension permanente ou prevailing tension dans la jurisprudence de la CEDH, comme

\footnotetext{
158 Opinion dissidente de M. le Juge Loucaides, Cour Européenne des Droits de l'Homme, arrêt du 21 novembre 2001, McElhinney c. Irlande, requête $\mathrm{N}^{\circ}$ 31253/96, \$ 4.

159 Opinion dissidente de M. le Juge Loucaides, Cour Européenne des Droits de l'Homme, arrêt du 21 novembre 2001, McElbinney c. Irlande, requête $\mathrm{N}^{\circ}$ 31253/96, \$ 3 .

160 Opinions dissidentes de M. M. les Juges Caflish, Cabral Barreto et Vajic, Cour Européenne des Droits de l'Homme, arrêt du 21 novembre 2001, McElhinney c. Irlande, requête No 31253/96, \1.

161 Cour Européenne des Droits de l'Homme, arrêt du 12 décembre 2002, Kalogeropoulou et autres c. Grèce et Allemagne, requête No 59021/00.
} 
le souligne le juge A. A. Cançado Trindade dans son vote dissident. ${ }^{162}$ On peut donc constater que la consécration de l'immunité de l'Etat est loin de refléter une vision pacifiée: tant la majorité de la CEDH que les opinions dissidentes de ses juges montrent que l'immunité de l'Etat est en plein processus d'évolution. Cette évolution avance paripassu avec la reconnaissance des droits des individus dans la sphère internationale, conduisant à la progressive érosion de la souveraineté de l'Etat.

Compte tenu de ce qui précède, l'analyse faite par la CIJ afin de vérifier s'il existe une coutume internationale tendant à relativiser l'immunité de l'Etat en cas de graves violations de Droits de l'Homme et du Droit Humanitaire s'avère largement critiquable. De fait, la pratique de l'Etat a donné des indices de l'apparition d'une nouvelle exception à l'immunité de l'Etat en ce qui concerne la violation de règles impératives, ce qui aurait dû être pris en compte dans la décision de la majorité.

\section{Conclusion}

Il peut être affirmé que la décision de la majorité dans l'affaire Allemagne c. Italie ne correspond pas aux développements du Droit International contemporain. En l'espèce, il y a un conflit évident entre l'immunité de l'Etat et de graves violations du Droit International Humanitaire ayant le statut de règle de jus cogens. L'argument de la CIJ fondé sur la distinction formelle entre des règles de procédure —immunité de l'Etat— et de fond — jus cogens — se révèle être totalement artificiel et incapable de garantir l'effectivité des règles de jus cogens violées. Il n'y a pas eu d'analyse des critères qui fondent cette distinction. La Cour a choisi de garder sa position d'arbitre neutre des controverses interétatiques, détachée des valeurs humanistes qui pourraient remettre en question sa légitimité. L'attitude formaliste de la Cour a écarté les obligations erga omnes issues de la règle de jus cogens violée, et a ignoré le droit à réparation des victimes, contrairement à sa jurisprudence dans les affaires Barcelona Traction et Timor Oriental. D'après le Projet d'articles sur la responsabilité des Etats, il serait évidemment contraire au Droit International de reconnaittre la licéité de la situation qui s'est créée en raison de la violation d'une règle impérative ou d'offrir de l'aide ou assistance

\footnotetext{
162 Opinion dissidente de M. le Juge A. A. Cançado Trindade, Cour Internationale de Justice, Immunités juridictionnelles de l'Etat (Allemagne c. Italie; Grèce intervenant), arrêt du 3 février 2012, \ 130 et suiv.
} 
afin de faire perdurer cette situation. Dans le cas concret, l'attribution de l'immunité à l'Allemagne constitue une grave violation de l'obligation de non-reconnaissance prévue à l'article 41 dudit projet.

La présomption selon laquelle les règles de jus cogens ne seraient pas applicables en matière civile peut également être mise en question. La nature des procédures — civile ou pénale — n'est donc pas pertinente et ne détermine pas les effets d'une règle de jus cogens par rapport à d'autres normes de Droit International telles que l'immunité de l'Etat. Les effets d'une norme impérative découlent de son essence, et ne sont pas rattachés à des conditions externes. L'argument selon lequel l'immunité, en tant que règle de procédure, devrait prévaloir sur l'accès à la justice, qui revêt également le caractère de norme procédure ne saurait non plus être défendu. En outre, l'immunité ne saurait constituer un obstacle irréductible à l'accès à la justice des victimes de graves violations de Droit International Humanitaire, surtout en cas d'absence de recours alternatif. De plus, si l'accès à la justice faisait partie des règles de jus cogens, comme cela a été reconnu par la Cour Interaméricaine des Droits de l'Homme, cela conduirait à le faire prévaloir sur les règles coutumières. L'immunité de l'Etat cèderait par conséquent, en faveur de la reconnaissance des droits individuels, conformément à la tendance du Droit International contemporain.

Même si cela n'était pas le cas, l'évolution même de la norme coutumière ne mènerait pas au résultat défini par la Cour. Le Droit International n'est pas statique, mais doit prendre en compte les transformations temporelles et l'évolution du système juridique. La thèse de l'immunité absolue à cédé la place à une immunité restreinte en raison des développements jurisprudentiels adaptés à un contexte spécifique. La jurisprudence a ancré la distinction entre jure imperii et jure gestionis, qui reflétait les priorités de la société compte tenu du rôle de l'Etat en tant que sujet de droit privé. Actuellement, avec la reconnaissance et l'évolution des Droits de l'Homme, du Droit International Humanitaire et du Droit Pénal International, la notion d'immunité est en train d'être réévaluée, car elle est devenue la source potentielle d'injustices. La distinction entre actes de puissance publique et actes de gestion est devenue obsolète, car elle n'englobe pas des situations dans lesquelles des Etats violent des normes impératives du Droit International. De grave crimes internationaux ne sauraient être considérés comme des fonctions typiques d'Etat afin d'entrer dans la catégorie des actes de puissance publique. Comme le souligne à juste titre M. le Juge A. A. Cançado Trindade dans son opinion dissidente dans la présente affaire, nous serions face à un delicta imperii. 
L'immunité souveraine, une notion définie par des avocats au XIX ${ }^{\text {ème }}$ siècle, a été utilisée par des Etats comme une façon de se soustraire à ses obligations de Droit International. ${ }^{163}$ Or, les Etats ne sauraient s'abriter derrière l'immunité afin d'échapper au respect des règles impératives du Droit International, en l'espèce, de graves violations du Droit Humanitaire. La raison d'Etat fait place à l'intérêt de l'humanité à assurer qu'il n'y ait pas d'impunité en cas de crimes internationaux. Il ne saurait être nié que l'attribution d'immunité génère une impunité, même si ce n'est qu'indirectement. La Cour a semblé reconnaittre dans l'affaire Allemagne c. Italie que les victimes pourraient difficilement faire valoir leurs droits auprès de tribunaux nationaux. On assiste actuellement surtout à une érosion progressive de la souveraineté étatique en faveur des droits individuels. Bien que l'immunité soit fondée sur la souverainté d'Etat et non pas sur l'idée de courtoisie et de dignité des nations, comme le défendent certains auteurs, l'idée même de souveraineté n'est pas absolue, comme en témoigne la notion de responsabilité de protéger, issue de l'interprétation téléologique de la Charte de l'ONu. La souveraineté implique la responsabilité de protéger la population mais n'inclut pas la pratique de crimes internationaux à l'encontre de la même population que celle qui devrait être protégée par un Etat territorial.

Les développement récents du Droit International renvoient à la reconnaissance d'une nouvelle exception à l'immunité de l'Etat, qui consiste en la violation de règles de jus cogens. La pratique de différents tribunaux commence à donner des indices d'un revirement de jurisprudence, en écartant l'immunité dans des situations impliquant des graves violations des Droits de l'Homme et du Droit International Humanitaire. Dans le cadre régional, bien que la CEDH reste réservée à ce sujet, certaines décisions ont été obtenues avec une faible majorité, les juges dissidents ayant souligné le besoin de limiter l'immunité de l'Etat en faveur de règles impératives. Cette tension permanente indique que l'immunité de l'Etat est en plein processus d'évolution, ce qui n'a pas été pris en compte par la Cour dans sa décision dans la présente affaire. Agissant de façon conservatrice, la Cour a choisi de préserver la stablité du système international et a dû

163 Wilberforce, Address to the Fifty-Eight Conference of the International Law Association, reprinted in International Law Association, Report of the Fifty-Eight Conference 513, 515 (1980), cité par Belsky, Merva \& Roht-Arriaza, op. cit., p. 365, note 2: l'immunité souveraine "is a concept devised by lawyers in the $19^{\text {th }}$ century [and] is now being used by nations generally as a technique for denying compliance with obligations". 
payer un prix élévé pour maintenir sa position. ${ }^{164}$ Elle s'est abstenue de mettre en équilibre des valeurs concurrentes, en ignorant les conséquences de sa décision sur la protection des victimes de sérieuses violations des Droits de l'Homme et du Droit Humanitaire qui avaient acquis le statut de jus cogens. Elle a seulement fait part de sa surprise et de son regret, en perdant l'opportunité de promouvoir une interprétation progressiste du Droit International, adaptée à la réalité actuelle. De manière peu surprenante, l'affaire Allemagne c. Italie met la CIJ à contre-sens de l'évolution du Droit International.

\section{Références}

\section{Livres et articles}

Badr, M., State immunity: an analytical and prognostic view, La Haye, 1984. Bartsch, K. \& Elberling, B., "Jus cogens vs. State immunity, round two: the decision of the European Court of Human Rights in the Kalogeropoulou et al. v. Greece and Germany decision", German Law Journal, 2003, 4, (5), pp. 477-491.

Belsky, A. C., Merva, M. \& Roht-Arriaza, N., "Implied waiver under the FSIA: a proposed exception to immunity for violations of peremptory norms of international law", California Law Review, 1989, 77, (2), pp. 365-415.

Bianchi, A., "Ferrini v. Federal Republic of Germany", The American Journal of International Law, 2005, 99, (1), pp. 242-248.

Bornkamm, C., "State immunity against claims arising from war crimes: the judgement of the International Court of Justice in jurisdictional immunities of the State", German Law Journal, 13, (6), pp. 773-782.

Boudreault, F., "Identifying conflicts of norms: the ICJ approach in the case of the jurisdictional immunities of the State (Germany v. Italy:

164 D'après l'opinion dissidente de M. M. les Juges Higgins, Kooijmans et Buergenthal, Cour Internationale de Justice, Mandat d'Arrêt du 11 avril 2000 (République Démocratique du Congo c. Belgique), arrêt du 14 février 2002, \5: "One of the challenges of present-day international law is to provide for stability of international relations and effective international intercourse while at the same time guaranteeing respect for human rights. The difficult task that international law today faces is to provide that stability in international relations by a means other than the impunity of those responsible for major human rights violations". 
Greece intervening)", Leiden Journal of International Law, 2012, (25), pp. 1003-1012.

Cançado Trindade, A. A., Tratado de direito internacional dos direitos humanos, Tome III, Ed. S. A. Fabris, Porto Alegre, Brasil, 2002.

Cançado Trindade, A. A., Evolution du droit international au droit des gens - L'accès des individus à la justice internationale, le regard d'un juge, Ed. Pedone, París, 2008.

Cançado Trindade, A. A., "Jus cogens: the determination and the gradual expansion of its material content in contemporary international case-law", en OEA, Comité Jurídico Interamericano, XXXV curso de derecho internacional (2008), Departamento de Derecho InternacionalSecretaría de Asuntos Jurídicos, Washington D.C., 2009, pp. 3-29.

Cançado Trindade, A. A., "Ampliación del contenido material del ius cogens", en OEA, Comité Jurídico Interamericano, XXXIV curso de derecho internacional (2007), Departamento de Derecho InternacionalSecretaría de Asuntos Jurídicos, Washington D.C., 2009, pp. 1-15.

Caplan, L. M., "State immunity, human rights and jus cogens: a critique of the normative hierarchy theory", American Journal of International Law, 2003, 97, pp. 741-781.

Cassese, A., International law, $2^{\mathrm{è}}$ éd., Oxford, 2005.

Cassese, A., "The character of the violated obligation", en Crawford, J., Pellet, A. \& Olleson, S., The law of international responsibility, Oxford University Press, 2010, pp. 415-420.

Cataldi, G., "The implementation of the ICJ's decision in the jurisdictional immunities of the State case in the Italian domestic order: what balance should be made between fundamental human rights and international obligations", ESIL Reflections, 2013, 2, (2), pp. 1-6.

Conforti, B., "The judgment of the International Court of Justice on the immunity of foreign States: a missed opportunity", The Italian Yearbook of International Law, 2012, XXI, (2011), pp. 135-142.

Cosnard, M., "Les immunités du chef d'Etat", in Colloque de la SFDI, Le chef d'Etat et le droit international, París, 2002, pp. 189-268.

Daillier, P., Forteau, M. \& Pellet, A., Droit international public, 8 è éd., LGDJ, París, 2009.

Dawidowicz, M., "The obligation of non-recognition of an unlawful situation", en Crawford, J., Pellet, A. \& Olleson, S., The law of international responsibility, Oxford University Press, 2010, pp. 677-686. 
De Sena, P. \& De Vittor, F., "State immunity and human rights: the Italian Supreme Court decision on the Ferrini case", The European Journal of International Law, 2005, 16, (1), pp. 89-112.

Espósito, C., "Jus cogens and jurisdictional immunities of States at the International Court of Justice: 'a conflict does exist", The Italian Yearbook of International Law, 2012, XXI, (2011), pp. 161-174.

Fox, H., "International law and restraints on the exercise of jurisdiction by national courts of States", en Evans, M. (dir.), International law,

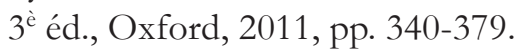

Fox, H., The law of State immunity, $2^{\grave{c}}$ éd., Oxford, 2008.

Johnson, T. A., "A violation of jus cogens norms as an implicit waiver of immunity under the Federal Sovereign Immunities Act", Maryland Journal of International Law, 1995, 19, (2), pp. 259-291.

Kessedjian, C., "Immunités", Répertoire Dalloz, de droit international, 2012.

Knuchel, S., "State immunity and the promise of jus cogens", Northwestern Journal of International Human Rights, 2011, 9, (2), pp. 149-183.

Lauterpacht, H., "The problem of jurisdictional immunities of foreign States", British Yearbook of International Law, 1951, (28), pp. 220-272.

Lecourt, R., L'Europe des juges, Bruylant, Bruselas, 2008 (réimpression de l'oeuvre publiée en 1976).

McGregor, L., "State immunity and jus cogens", The International and Comparative Law Quarterly, 2006, 55, (2), pp. 437-445.

Ménard, M., "Circumstances precluding wrongfulness in the ILC articles on State responsibility: compliance with peremptory norms", en Crawford, J., Pellet, A. \& Olleson, S., The law of international responsibility, Oxford University Press, 2010, pp. 449-453.

Moneta, F., "State immunity for international crimes: the case of Germany versus Italy before the ICJ", The Hague Justice Portal, pp. 1-8, en www. haguejusticeportal.net

Orakhelashvili, A., "State immunity and international public order", German Yearbook of International Law, 2002, 45, pp. 227-267.

Orakhelashvili, A., "State immunity and hierarchy of norms: why the House of Lords got it wrong", European Journal of International Law, 18, (5), pp. 955-970.

Orakhelashvili, A., Peremptory norms in international law, Oxford, 2006.

Pavoni, R., "An American anomaly? On the ICJ's selective reading of United States practice in jurisdictional immunities of the State", The Italian Yearbook of International Law, 2012, XXI, (2011), pp. 143-159. 
Reimann, M., "A human rights exception to sovereign immunity: some thoughts on Princz v. Federal Republic of Germany", Michigan Journal of International Law, 1995, 16.

Sossai, M., "Are Italian courts directly bound to give effect to the jurisdictional immunites judgement?", The Italian Yearbook of International Law, 2012, XXI, (2011), pp. 175-189.

Talmon, S., "Jus cogens after Germany v. Italy: substantive and procedural rules distinguished", Leiden Journal of International Law, 2012, (25), pp. 979-1002.

Tomuschat, C., "The international law of State immunity and its development by national institutions", Vanderbilt Journal of Transnational Law, 44, pp. 1105-1140.

Trapp, K. N. \& Mills, A., "Smooth runs the water where the brook is deep: the obscured complexities of Germany v. Italy", Cambridge Journal of International and Comparative Law, 2002, 1, pp. 153-168.

Van Alebeek, R., The immunities of States and their officials in international criminal law and international human rights law, Oxford, 2008.

Verhoeven, J. (dir), Les droit international des immunités: contestation ou consolidation ?, París, 2004.

Verhoeven, J., "Les immunités propres aux organes ou autres agents des sujets de droit international", en Verhoeven, J. (dir.), Le droit international des immunités: contestation ou consolidation ?, París-Bruselas, 2004, pp. 61-146.

Virally, M., "Réflexions sur le jus cogens", AFDI, 1966, 12, pp. 5-29.

Visscher, C., "Positivisme et jus cogens", RGDIP, 1971, 75, pp. 5-11.

Watt, H. M., "Les droits fondamentaux devant les juges nationaux à l'épreuve des immunités juridictionnelles: à propos de l'arrêt de la Cour internationale de justice, immunites juridictionnelles de l'Etat (Allemagne c. Italie; Grèce intervenant), du 3 février 2012", Revue critique de droit international privé, 2012, 101, (3), pp. 539-552.

Watt, H. M., "Une perspective internationaliste-privatiste", en Verhoeven, J. (dir.), Le droit international des immunités: contestation ou consolidation?, París-Bruselas, 2004, pp. 265-274.

Wickremasinghe, C., "Immunities enjoyed by officials of states and international organizations", en Evans, M. (ed.), International law, $3^{\grave{e} e ́ d ., ~}$ Oxford, 2011, pp. 380-410.

Zimmermann, A., "Sovereign immunity and violations of international jus cogens. Some critical remarks", Michigan Journal of International Law, 1995, 16. 


\section{Cours de l'Académie de Droit International de la Haye}

Cançado Trindade, A. A., "international law for humankind: towards a new jus gentium (I) - General course on public international law", RCADI, 2005, 316, pp. 9-439.

Dunbar, N. C. H., "Controversial aspects of sovereign immunity in the case law of some States", RCADI, 1971, 132, pp. 197-362.

Gaja, G., "Jus cogens beyond the Vienna Convention", RCADI, 1981, 172, (III), pp. 271-316.

Sinclair, I., "The law of sovereign immunity: recent developments", RCADI, 1980, 167, pp. 113-284.

Trooboff, P. D., "Foreign State immunity: emerging consensus on principles", RCADI, 1986, 200, pp. 235-432.

Watts, A., "The legal position in international law of heads of States heads of governments and foreign ministers", RCADI, 1994, 247, pp. 10-130.

Weiss, A., "Compétence ou incompétence des tribunaux à l'égard des Etats étrangers", RCADI, 1923, 1, pp. 521-552.

\section{Jurisprudence}

\section{Cour Permanente de Justice Internationale et Cour Internationale de Justice}

Cour Internationale de Justice, Activités armées sur le territoire du Congo (2002) (République Démocratique du Congo c. Rwanda), compétence de la Cour et recevabilité de la demande, arrêt du 3 février 2006.

Cour Internationale de Justice, Activités militaires et paramilitaires an et contre le Nicaragua (Nicaragua c. Etats-unis d'Amérique), arrêt du 27 juin 196 (fond).

Cour Internationale de Justice, Affaire relative an personnel diplomatique et consulaire des Etats-Unis à Tébéran (Etats-Unis d'Amérique c. Iran), demande d'indication de mesures conservatoires, arrêt du 15 décembre 1979.

Cour Internationale de Justice, Barcelona Traction Light and Power Company Limited (Belgique c. Espagne), arrêt du 5 février 1970.

Cour Internationale de Justice, Conséquences juridiques de l'édification d'un mur dans le territoire palestinien occupé, avis consultatif du 9 juillet 2004. 
Cour Internationale de Justice, Conséquences juridiques pour les Etats de la présence continue de l'Afrique du Sud en Namibie (Sud-ouest africain) nonobstant la résolution 276 (1970) du Conseil de Sécurité, avis consultatif du 21 juin 1971.

Cour Internationale de Justice, Détroit de Corfou (Royaume-Uni c. Albanie), arrêt du 9 avril 1949 (fond).

Cour Internationale de Justice, Immunités juridictionnelles de l'Etat (Allemagne c. Italie; Grèce intervenant), arrêt du 3 février 2012.

Cour Internationale de Justice, Licéité de la Menace on de l'Emploi d'Armes Nucléaires, avis consultatif du 8 juillet 1996.

Cour Internationale de Justice, Mandat d'Arrêt du 11 avril 2000 (République Démocratique du Congo c. Belgique), arrêt du 14 février 2002.

Cour Internationale de Justice, Plateau Continental (Jamahiriya arabe libyenne c. Malte), arrêt du 21 mars 1984.

Cour Internationale de Justice, Plateau continental de la mer du Nord (République Fédérale d'Allemagne c. Danemark); (République Fédérale d'Allemagne c. Pays-Bas), arrêt du 20 février 1969.

Cour Internationale de Justice, Réserves à la Convention pour la Prévention et la Répression du Crime de Génocide, avis consultatif du 28 mai 1951.

Cour Internationale de Justice, Timor oriental (Portugal c. Australie), arrêt du 30 juin 1995.

Cour Permanente de Justice Internationale, Usina de Chorzón, arrêt du 26 juillet 1927 (compétence), Série A No 9.

\section{Cour Européenne des Droits de l'Homme}

Cour Européenne des Droits de l'Homme, arrêt du 18 février 1999, Waite et Kennedy c. Allemagne, requête No 26083/94.

Cour Européenne des Droits de l'Homme, arrêt du 18 février 1999, Beer et Regan c. Allemagne, requête No 28934/95.

Cour Européenne des Droits de l'Homme, arrêt du 21 novembre 2001, McElhinney c. Irlande, requête $\mathrm{N}^{\mathrm{o}} 31253 / 96$.

Cour Européenne des Droits de l'Homme, arrêt du 21 novembre 2001, Fogarty c. Royaume-Uni, requête $\mathrm{N}^{\circ}$ 37112/97.

Cour Européenne des Droits de l'Homme, arrêt du 21 novembre 2001, Al-Adsani c. Royaume-Uni, requête No 35763/97.

Cour Européenne des Droits de l'Homme, arrêt du 12 décembre 2002, Kalogeropoulou et autres c. Grèce et Allemagne, requête No 59021/00. 


\section{Cour Interaméricaine des Droits de l'Homme}

Cour Interaméricaine des Droits de l'Homme, avis consultatif No 18/03 du 17 septembre 2003, Condition Juridique des Immigrants sans-papiers, Série A No 18.

Cour Interaméricaine des Droits de l'Homme, Goiburú et autres c. Paraguay, décision du 22 septembre 2006.

Cour Interaméricaine des Droits de l'Homme, La Cantuta c. Pérou, décision du 29 novembre 2006.

\section{Tribunal Pénal International pour l'ex-Yougoslavie}

Tribunal Pénal International pour l'ex-Yougoslavie, Chambre de Première Instance, décision du 10 décembre 1998, Procureur contre Anto Furundzija, IT-95-17/1-T. 